Chapter 2

\title{
Decision Support Systems in Medicine - Anesthesia, Critical Care and Intensive Care Medicine
}

\author{
Thomas M. Hemmerling, Fabrizio Cirillo and \\ Shantale Cyr \\ Additional information is available at the end of the chapter \\ http://dx.doi.org/10.5772/51756
}

\section{Introduction}

A decision support system (DSS) in medicine is a software designed to assist the medical team in the decision making process; it deals with organizational, diagnostic and therapeutic problems, using data (e.g. variables of the patient) as inputs to combine with models and algorithms giving advice in form of monitor alerts, color codes, or visual messages; it does not replace the human operator, but can improve the quality of care. Modern society more and more asks the medical community for 'infallibility' in clinical practice, but errors is part of human intervention: emotions, behavioral and psychological patterns, or difficult contexts can influence human performances. For humans, it is simply impossible to recall all diagnostic and therapeutic options at any time for any given patient [1]. The use of DSSs in the clinical management could solve this problem helping specialists with diagnostic or therapeutic suggestions, making it easier to follow validated guidelines, reducing the incidence of faulty diagnoses and therapies [2], and changing incorrect behaviors.

Early computerized medical systems date back to the early 60ies [3]. First prototypes were used to train medical students in establishing a diagnosis [4]. The evolution of these systems has followed the general innovation in technology and their capacities constantly increase over time, from only educational tools to intelligent systems for patient management.

Basically, a DSS can be designed using knowledge representation, in the form of clinical algorithms, mathematical pathophysiological models, Bayesian statistical systems and diagrams, neural networks, fuzzy logic theories, and symbolic reasoning or "expert" systems [5]. A DSS has to be conceived suitable and user-friendly; the 'rules structure' should be 
easily understood, the rules process should be intuitive and open for collaboration, all decisions should be reproducible and the user interface easy to use (Figure 1) [6].

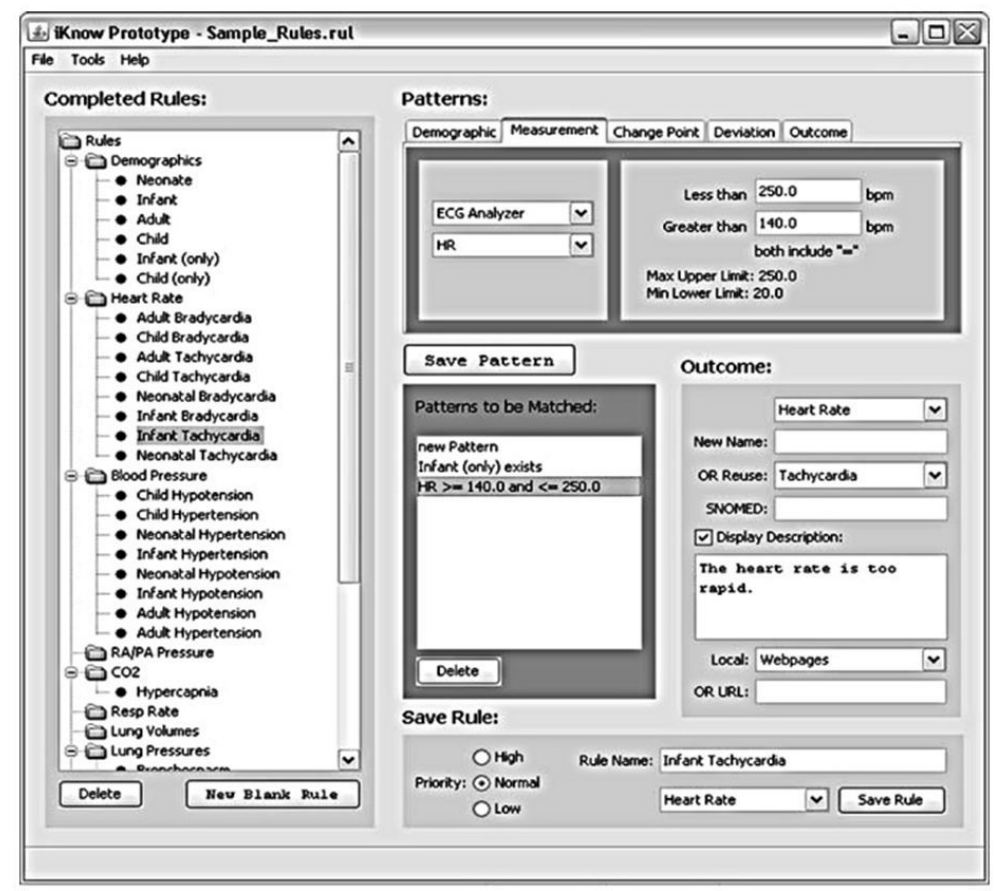

Figure 1. Graphical user interface [6].

DSSs in medicine could play a role in every field: a modern DSS is conceived to predict rehabilitation protocol for patients with knee osteoarthritis [7]. Another example of a modern DSS is a system that uses anthropometric information and questionnaire data to predict obstructive sleep apnea [8]. The use of DSSs has been proposed to treat major depression [9]; a DSS has been validated recently to diagnose the common flu [10]; a DSS has been developed to support the treatment of epilepsy [11]. Another DSS has been presented in the field of gynecology [12].

At present, it is not clear if an improvement of medical performance can always be transferred into an improvement of patient outcomes [13,14] [15], and although better adherence to guidelines is proven, this cannot always be translated into abandoning habits of wrong-doing [16]. Furthermore, there are some considerable barriers to the widespread diffusion of these systems, like costs, cultural issues and lack of standards [2] [17] [18].

These systems are usually produced with limited private funds; mass production is limited by economic pressures. Lack of standardization often represents a "political" problem. There are always emotional barriers for physicians and other health care providers to 'rely' on the help of devices in order to make proper decision. 
Anesthesiologists and critical care specialists are very involved in patient safety; excellence in their fields needs a collection of nontechnical, nonclinical skills that may be classified as "task management", "team working", "situation awareness", and "decision-making"[19]. Developing information and decision technology support systems for these skills also means to significantly improve the quality, flow, and efficiency of medical performance [20].

This chapter will focus on DSSs for anesthesiologists and critical care specialists in different areas: perioperative management, the emergency and intensive care medicine.

\section{Decision support systems for anesthesia in the operating room}

Anesthesiologists in the operating room have to provide direct patient care. Anesthesiologists are considered the "pilots of human biosphere" [21], and terms like "takeoff" and "landing" for the process of inducing anesthesia and reversing it, are very common; since these are the two dominant and critical moments of anesthesia, often, maintenance of anesthesia receives less attention [22]. To assure safe and good patient care during the surgical procedure, an anesthesiologist interacts with several devices: he becomes "the mediator between patient and machine while the machine is mediating between patient and anesthesiologist; all are hybrids in action and each is unable to act independently" [22]. It is impossible to consider the anesthetic work without machines just as it is impossible to imagine a pilot without his joysticks, buttons and computers.

Decision support systems for anesthesia in the milieu of the operating room are software shaped to assist the anesthesiologist in his difficult work during the surgical procedure. Let's divide DSSs for anesthesia in the operating room into three classes: DSSs designed for perioperative use, DSSs for one single intraoperative problem (simple DSSs) and DSSs for multiple problems (complex DSSs).

\subsection{Organizational DSSs and implementation in AIMS in the perioperative context}

In his everyday activity, the anesthesiologist deals not only with patient-related issues, but also with many kinds of organizational problems, like strictly hierarchical command structures or deficits in providing important drugs or devices that can cause serious accidents. Reason [23] has proposed a scheme of the development of an organizational accident (Figure 2).

It is not possible to consider the anesthesiologist's responsibility only during the surgical intervention; as a pilot has to control his systems before the flight, anesthesiologists must continuously assess the patient status, from pre-operative assessment till post-operative care. As a 'commander-in-chief', he has to make the final check of everything 'anesthetic' in the operating room, despite the presence of nurses or respiratory technicians. One type of DSS can deal with organizational problems in order to prevent accidents.

The first example of how DSSs may improve safety in the operating environment is a DSS whi generates dynamically configured checklists for intraoperative problems [24]. It is interesting that the database built with 600 entries of two anesthesia textbooks and organ- 
ized in problems and corresponding abnormalities, considers also technical hitches, like e.g. inefficiency in anesthesia machines or incorrect position of an endotracheal tube. For each abnormality detected by monitors and confirmed by the practitioner, the software formulates a list of questions, starting with a recognized "high-impact abnormality" (every abnormality uniquely associated with a problem); questions about the "high-impact abnormality" are presented to users as closed-type questions, i.e. they can be answered as "yes" or "no", to facilitate a quick response.

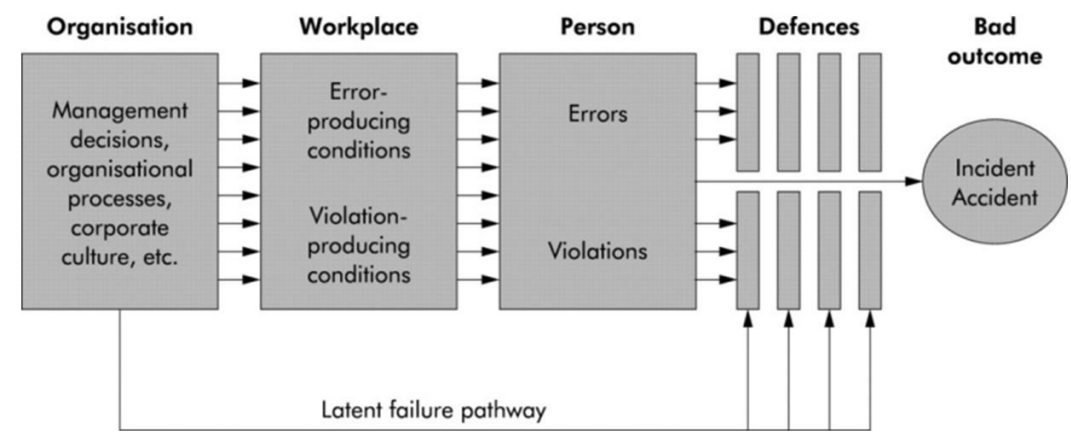

Figure 2. The development of an organizational accident [23].

Preoperative tests are crucial for the stratification of the anesthetic risk, for the choice of the anesthesia technique but also to define the anesthesiologist's behavior. A Canadian group [25] found that the mean cost of investigations was reduced from $\$ 124$ to $\$ 73$ if data for patients were assessed by staff anesthesiologists. Another study [26] demonstrated that, following definite preoperative diagnostic guidelines, possible savings per 1000 patients would be $€ 26287$ and $€ 1076$ if duplicated tests were avoided.

A DSS for this purpose, the System for Pre-Operative Test Selection (SPOTS), has been developed to assist physicians in selecting the right preoperative, individualized and clinically relevant tests [27]. The software uses a database comprising of patient data, clinical history, a list of surgical procedures, standard guidelines for preoperative investigations, type and cost of investigations, and investigation results: the DSS then suggests the tests and performs a cost comparison.

Airway management represents one of the most important challenges for the anesthesiologist. The main causes of anesthesia-related mortality are respiratory and cardiocirculatory events [28, 29]. One of the most important aims of preoperative assessment is predicting a difficult intubation; it means to timely prepare airway devices to facilitate a possibly difficult procedure. Currently, the gold standard for the evaluation of the difficulty of intubation is the Cormack and Lahane classification, but it's feasible only through direct laryngoscopy. A DSS for estimating the Cormack classification was presented in 2009 [30]; it was based on data of 264 medical records from patients suffering from a variety of diseases. It used 13 ba- 
sic anthropometrical features (Figure 3) to predict easy (Cormack I and II) or difficult intubation (Cormack III and IV). The system showed an average classification accuracy of $90 \%$.

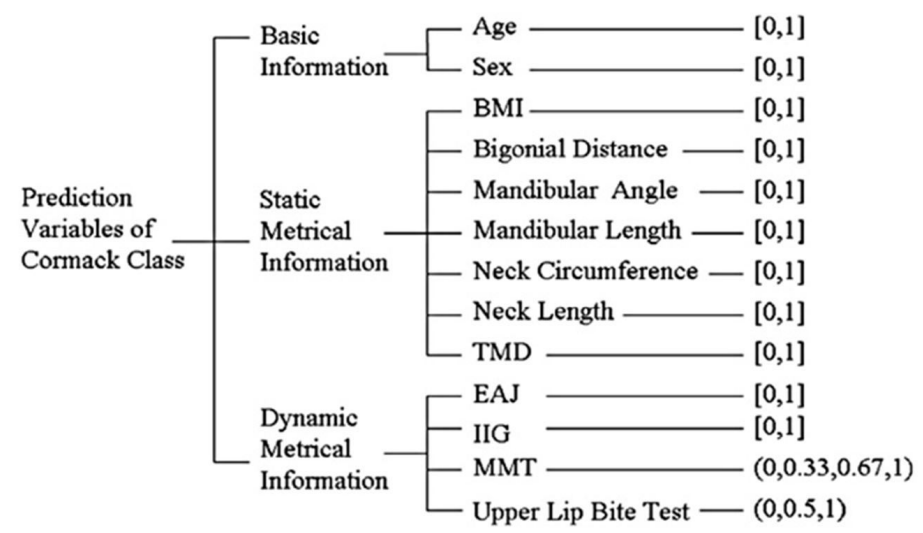

Figure 3. The 13 variables for Cormack classification and their encoding schemes. BMI, for body mass index; TMD, tyro-mental distance; EAJ, atlanto-axial joint; IIG, interincisor gap; MMT, modified Mallampati test. Binary values $(0,1)$ were used for variables with only two attributes. Values as 0, 0.5 and 1 were used for variables with three attributes. Values as $0,0.33,0.67,1$, were used for variables with four attributes [30].

Anesthesia information management systems (AIMS) can reduce the anesthesiologist's workload. Implementation of DSSs in AIMS represents a natural evolution of information technology: DSSs can use data stored in AIMS to give diagnostic or therapeutic messages. This development increases the usefulness of both systems [31, 32].

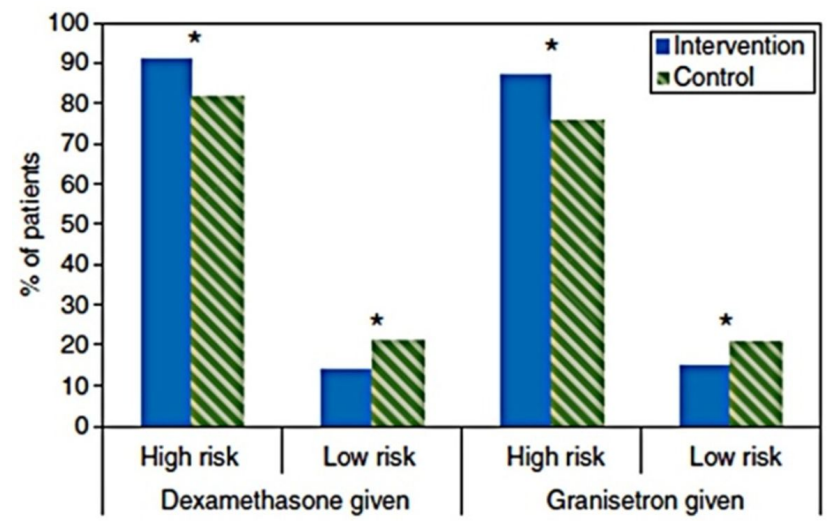

Figure 4. Percentage of patients involved in prophylaxis. *Statistically significant difference [33].

A recent example of how a DSS combined with an AIMS can improve performance and outcomes is shown in a study about automated reminders for prophylaxis of postopera- 
tive nausea and vomiting (PONV) [33]. A database was implemented with PONV prophylaxis guidelines. The comparison of two groups (one with only AIMS and the other with AIMS and also DSS), found that automated reminders were more effective for adherence to PONV prophylaxis (Figure 4).

It also showed a reduction of inappropriate administration of PONV prophylaxis medication to low-risk patients: automated reminders not only are effective in promoting correct actions, but may also prevent unnecessary prescription of medication, hence reducing drug costs. Although the DSS significantly improved adherence to the PONV guidelines, guidelines adherence decreased to the level before use of the DSS after its withdrawal from clinical routine (Figure 5).

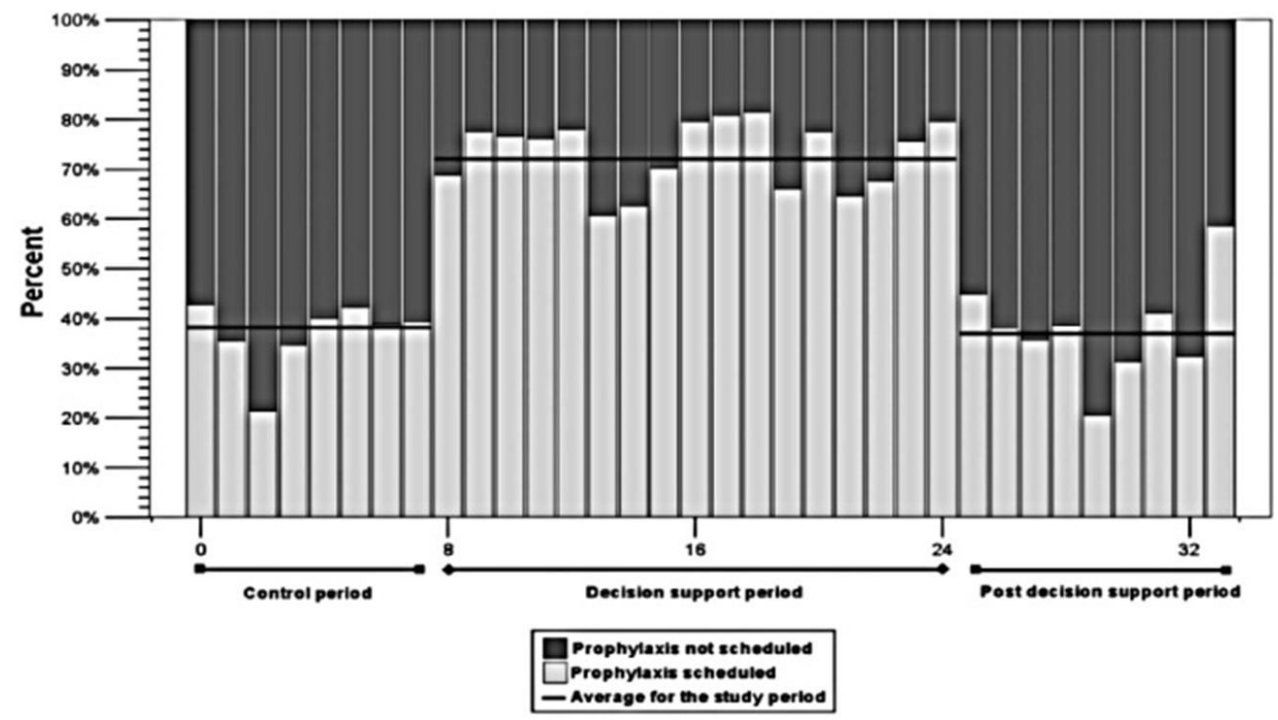

Figure 5. Guidelines adherence for high risk patients by week [16].

Surgical wound infections are relatively common, as they are considered the second most common complications occurring in a hospitalized patient [34, 35], and the second most common nosocomial infections, occurring in $2 \%-5 \%$ of surgeries and in up to $20 \%$ of abdominal surgeries [36]. They have a significant economical impact, because patients affected spend more time in the hospital and are more in danger to be admitted to an intensive care unit, to be readmitted to the hospital after discharge, or to die [37]. Antimicrobial prophylaxis is most effective when administered before surgical incision, with an optimal time to be within 30 minutes before incision or within 2 hours if vancomycin is administered [38, 39].

In order to facilitate timely administration, DSSs were implemented in AIMS to obtain better adherence with those guidelines. One of these is an automated computer-based documentation that generates automatic reminders to the anesthesia team and the surgeon[40]. In this 
study, authors found that $70 \%$ of all surgical patients received their antibiotics within $60 \mathrm{~min}$ of incision (Figure 6); after one year, the adherence increased to about $92 \%$.

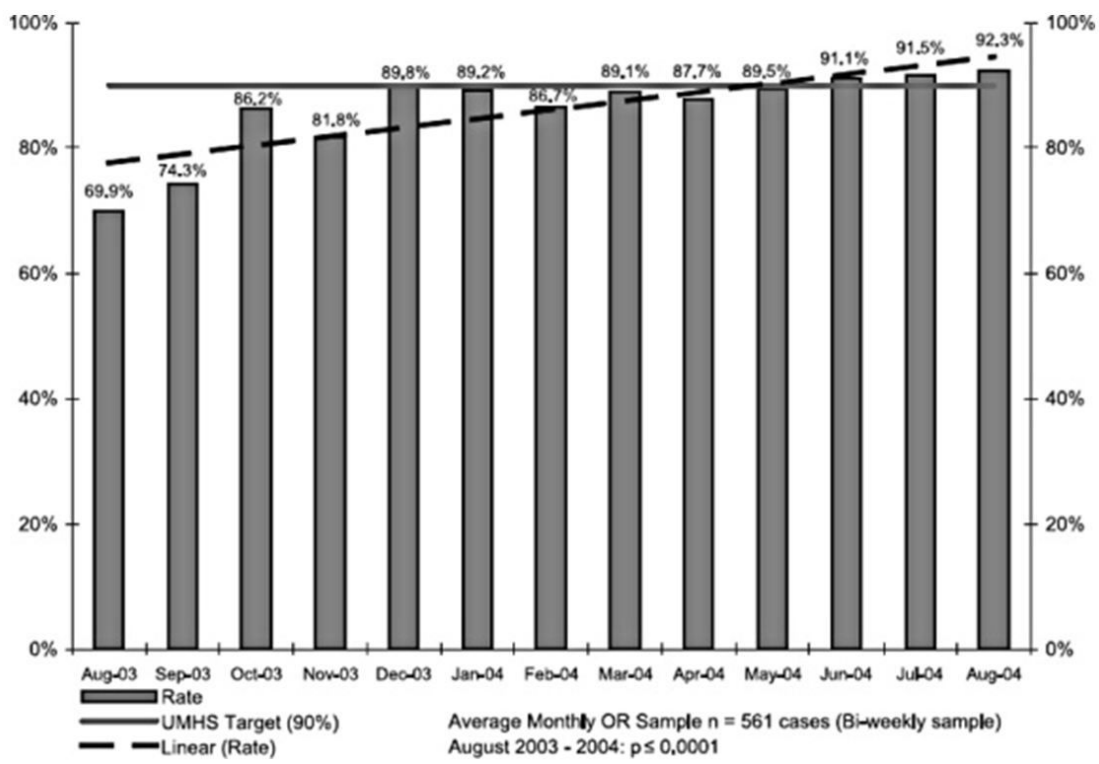

Figure 6. Administration of antibiotic: gradually increasing to about 92\% [40].

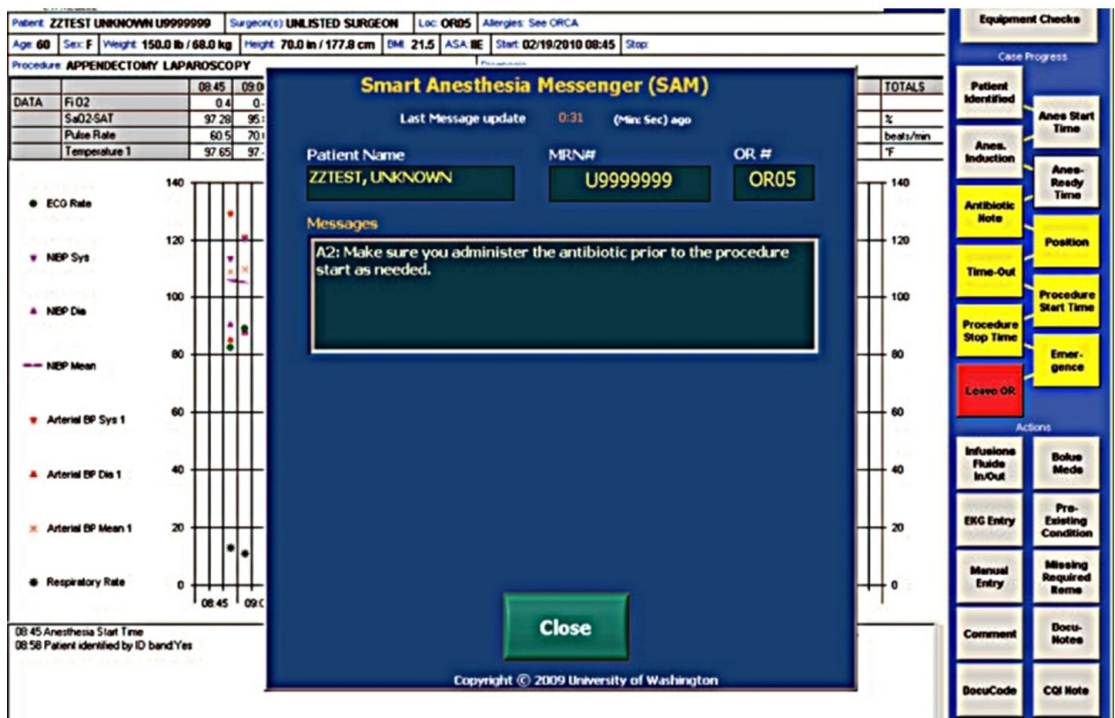

Figure 7. Anesthesia information management system screen overlaid by SAM screen [41]. 
Another DSS for antibiotic prophylaxis, the so-called Smart Anesthesia Messenger (SAM) [41], analyzes AIMS documentation data in real-time. Conceived as the final stage of intervention, after implementation in an AIMS, SAM transmits reminder messages to the AIMS screen to improve compliance of antibiotic administration before surgical incision (Figure 7). The addition of real-time reminders and feedback via SAM achieved near $100 \%$ compliance.

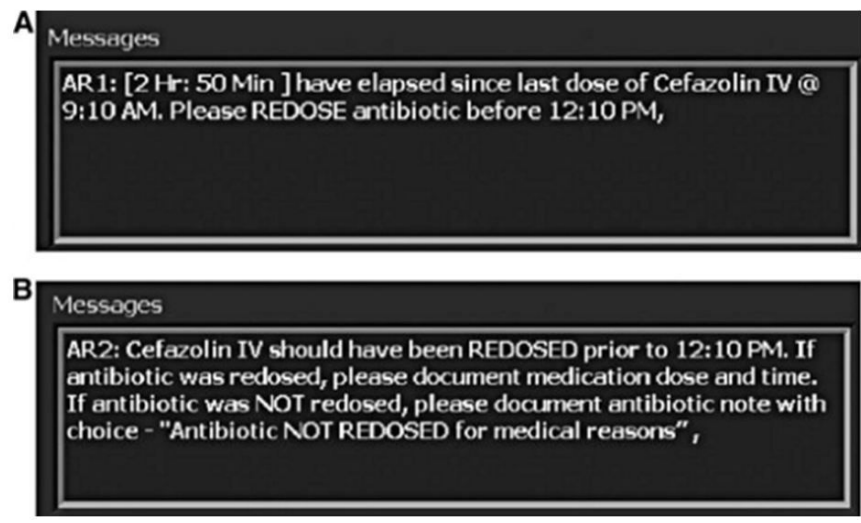

Figure 8. Messages for antibiotic re-dose. (A) Message reminding anesthesia team about need for re-dose. (B) Message about documenting re-dose [42].

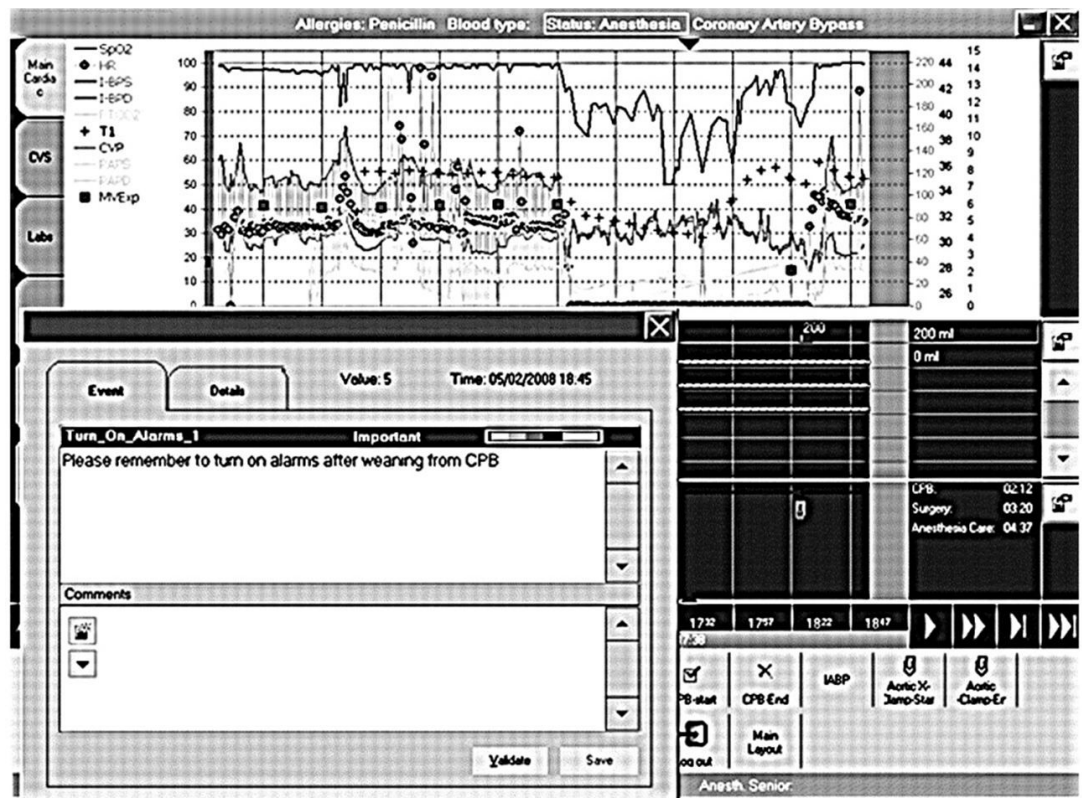

Figure 9. Main window of anesthesia information management system with the electronic reminder [45]. 
A follow-up study investigated the impact of the same DSS on the re-dosing of antibiotic therapy [42] in comparison with the use of only AIMS. Re-dosing could be important to maintain the necessary serum concentration of drug, to reduce the risk of postoperative wound infections in procedures that exceed of two half-lives of an antibiotic drug [43, 44]. In this study, a reminder message of re-dosing was effectuated every 3 hours (the shortest re-dose interval in guidelines of University of Washington Medical Center). The SAM detected the eventual administration of the prophylactic antibiotic drug, if necessary, it triggers an internal timer specific to that antibiotic and generates reminder icons $15 \mathrm{~min}$ prior to the time of re-dosing; these messages are repeated every 6 minutes until the dose is administered and documented (Figure 8). The employment of real-time decision support improved the success rate to $83.9 \%$.

A further example of advantageous use of DSS integrated in AIMS is an electronic reminder to switch on the ventilator alarms after separation from cardiopulmonary bypass (CPB) [45]. In cardiac surgery, during the CPB period, monitor alarms are often disabled; the alarms are frequently not reactivated. The software detects the separation from CPB by return of aortic and pulmonary blood flow, the resumption of mechanical ventilation and the reappearance of end-tidal $\mathrm{CO}_{2}$. If alarms have not been reactivated after the separation from $\mathrm{CPB}$, an electronic reminder appears on the AIMS screen (Figure 9). The alarm reactivation increased from $22 \%$ to $83 \%$.

\subsection{Simple DSSs for a single intraoperative problem}

A simple DSS combines a small amount of data to deal with one particular problem; it is like an electronic textbook about a specific issue, with the capability of giving the important information at the right time. Usually, problems for which these DSSs are created are very common or insidious. A simple DSS could represent the first step for the progressive development of a more complex DSS.

An example of a simple DSS is a system that detects 'light' anesthesia using as input the changes of mean arterial pressure (MAP) [46]. Krol and Reich considered a $12 \%$ change in median MAP in comparison with the median value of MAP over the previous $10 \mathrm{~min}$ period a parameter to trigger warnings for recognition of light anesthesia.

Another DSS involved in the detection of light anesthesia is an algorithm that relates different MAC values of volatile anesthetics to different intravenous sedative or hypnotics agents administered at the same time [47].

The introduction of fuzzy logic for setting up DSSs is founded on the ability of fuzzy-logic in dealing with the incompleteness and vagueness that often characterize medical data and knowledge [3]; in 1997, a fuzzy-logic based DSS to control the supply of oxygen in a patient during low-flow/closed-loop anesthesia was presented (Figure 10) [48].

A more recent Fuzzy-Logic Monitoring System (FLMS) has been developed [49]; this is a DSS conceived to detect critical events during anesthesia; it is able to detect only hypovolemia, using as inputs heart rate (HR), blood pressure (BP) and pulse volume (PV). Hypovolemia is classified as mild, moderate or severe. The FLMS was evaluated in 15 patients 
using off-line data and was found to be in good agreement with the anesthetist's diagnosis. An upgrading of this system, FLMS-2 [50], tested in 20 off-line patients, has demonstrated a sensitivity of $94 \%$, specificity of $90 \%$ and predictability of $72 \%$. The user interface of FLMS-2 is shown in Figure 11.

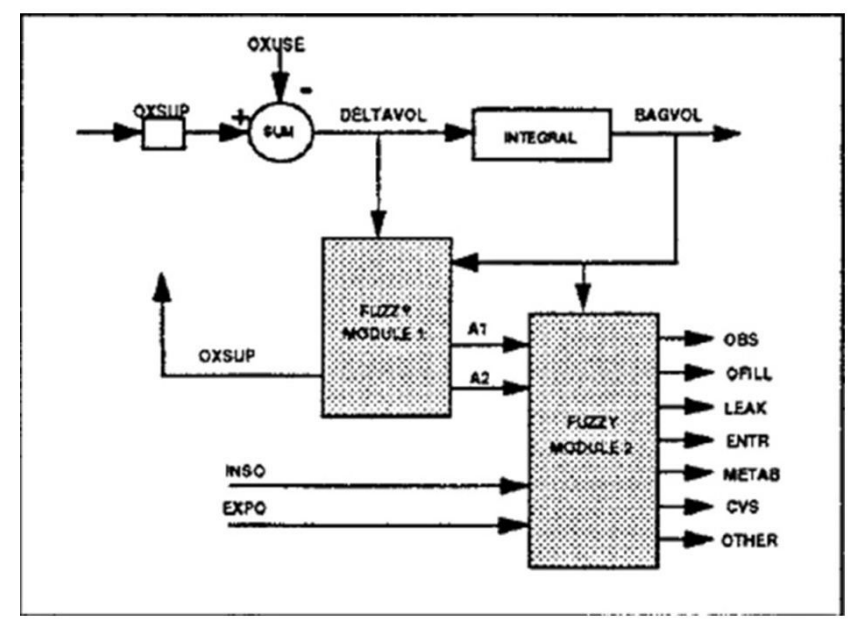

Figure 10. Scheme of fuzzy logic control system. Volume of the reservoir bag (BAGVOL) and his rate of change (DELTAVOL) are the inputs data for the first module (FZ module 1) to calculate the supply of oxygen (OXSUP); this value is sent as output data together with generated alarms ( $A L 1$ and $A L 2$ ) to the second module (FZ module 2), that correlates them with oxygen concentration values in inspired (INSO) and expired air (EXPO) to generate simple diagnostic messages including obstructions (OBS), overfilling (OFILL), leakage (LEAK), and entrapment ((ENTR) in the system and metabolism (METAB), cardiovascular (CVS) and other (OTHER) problems with patient [48].

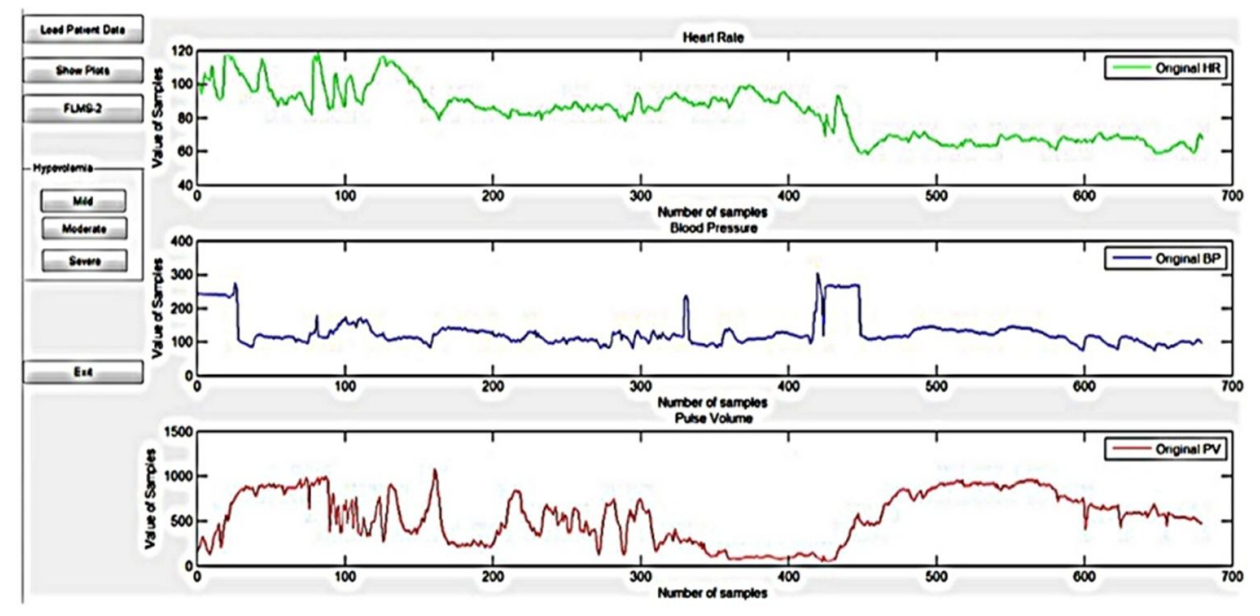

Figure 11. Graphic user interface [50]. 


\subsection{Complex DSSs for intraoperative use}

A complex DSS is software dealing with multiple problems. According with the complexity of the issue, it usually requires the collection of a certain number of information to combine with mathematical algorithm. It does not respond only to one problem, but can recognize different questions, sometimes inherent in a same category. These systems have to be considered as "intelligent textbooks".

One of the first complex DSSs for critical events in anesthesia was SENTINEL [51]. Based on fuzzy logic templates, this system used signals to establish a diagnosis despite missing information: it calculated the impact of lack of one or more signals for a certain condition via the estimation of the completeness factor [52]; the combination of some signals was judged as more important than others. The likelihood of a given diagnosis is measured considering two parameters of evidence: the belief (total of data supporting the evidence of a diagnosis) and the plausibility (the amount of data that do not contradict the diagnosis). At the beginning, this system was designed to detect only one problem, malignant hyperpyrexia ( $\mathrm{MH}$, between 1:5000 and 1:100000 episodes [53]). Lowe and Harrison [54] set up rules based on characteristic patterns of changes in heart rate, end-tidal carbon dioxide and temperature found in the literature and tested their software in a human simulator (Human Patient Simulator, version 1.3, University of Florida). During open surgery, the algorithm detected MH 10 minutes before the anesthetist; during laparoscopic surgery, in a condition with some similarities to $\mathrm{MH}$ (high end tidal $\mathrm{CO}_{2}$, cardiovascular changes), the diagnosis was only transient. Afterwards, SENTINEL was implemented with other rules to deal with other six conditions (Table 1). The interface of the system is depicted in Figure 12. SENTINEL was only tried in off-line tests, and its diagnostic alarms were compared with the annotations of anesthetists, showing a sensitivity of $95 \%$ and a specificity of $90 \%$ (during the period between induction and recovery phases).

\begin{tabular}{|c|c|}
\hline Problem & Description \\
\hline Inadequate analgesia (IA) & $\begin{array}{l}\text { Significant increases in heart rate and systolic blood pressure over } \\
\text { about half a minute; fall in pulse volume over the same period }\end{array}$ \\
\hline Malignant hyperpyrexia (MH) & $\begin{array}{l}\text { Significant rises in heart rate and end-tidal } \mathrm{CO}_{2} \text { concentration over } \\
\text { a number of minutes, followed by low peripheral saturation and a } \\
\text { high core body temperature }\end{array}$ \\
\hline $\begin{array}{l}\text { Increased intracranial pressure } \\
\text { (IICP) }\end{array}$ & $\begin{array}{l}\text { Short term decrease in heart rate and simultaneous rise in systolic } \\
\text { blood pressure }\end{array}$ \\
\hline Pulmonary shunt (PS) & Low peripheral saturation \\
\hline Cardiac output failure (COF) & $\begin{array}{l}\text { Sudden drop in end-tidal } \mathrm{CO}_{2} \text { concentration and systolic blood } \\
\text { pressure; followed by desaturation }\end{array}$ \\
\hline $\begin{array}{l}\text { Absolute hypovolaemia } \\
\text { (AHV) }\end{array}$ & $\begin{array}{l}\text { Fall in systolic blood pressure, and pulse volume; corresponding } \\
\text { increase in heart rate }\end{array}$ \\
\hline Relative hypovolaemia (RHV) & $\begin{array}{l}\text { Fall in systolic blood pressure; increase in pulse volume and heart } \\
\text { rate }\end{array}$ \\
\hline
\end{tabular}

Table 1. Diagnoses and their descriptions for the fuzzy trend templates [52]. 


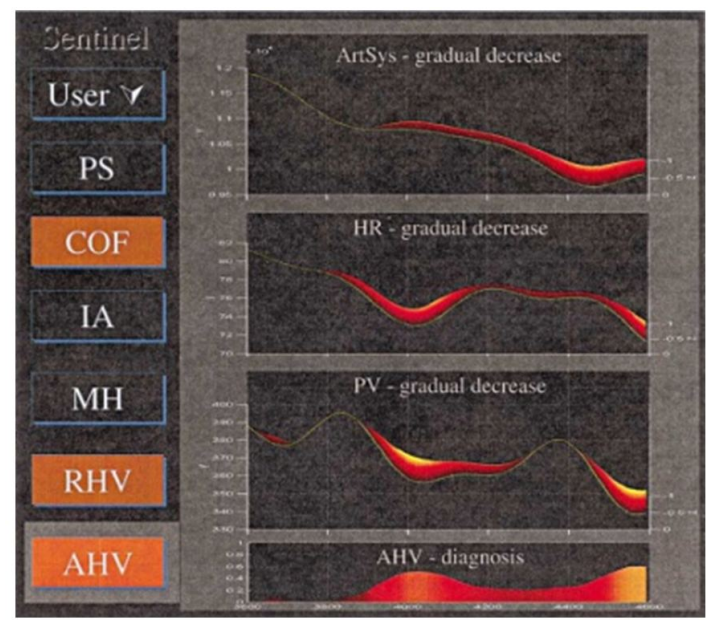

Figure 12. Prototype of SENTINEL user interface. Weak diagnosis of absolute hypovolemia (AHV) [55].

In the wake of SENTINEL, another DSS for critical events in anesthesia was presented in 2007, called Real Time-Smart Alarms for Anesthesia Monitoring (RT-SAAM) [56]. Initially, it was proposed to recognize and suggest treatment options of hypovolemia and decreasing cardiac output. Based on the evidence that hypovolemia can be detected by monitoring systolic pressure variations (SPV) in patients artificially ventilated [57], the DSS filtered the blood pressure $(\mathrm{BP})$, pulse volume $(\mathrm{PV})$, end-tidal carbon-dioxide $\left(\mathrm{ETCO}_{2}\right)$ waveforms and calculated the SPV and the absolute PV values, providing diagnostic information on the monitor in real-time (Figure 13). Tested in 18 patients in retrospective tests and in 8 patients during real-time tests, a moderate level of agreement between the DSS and the anesthesiologist was determined.

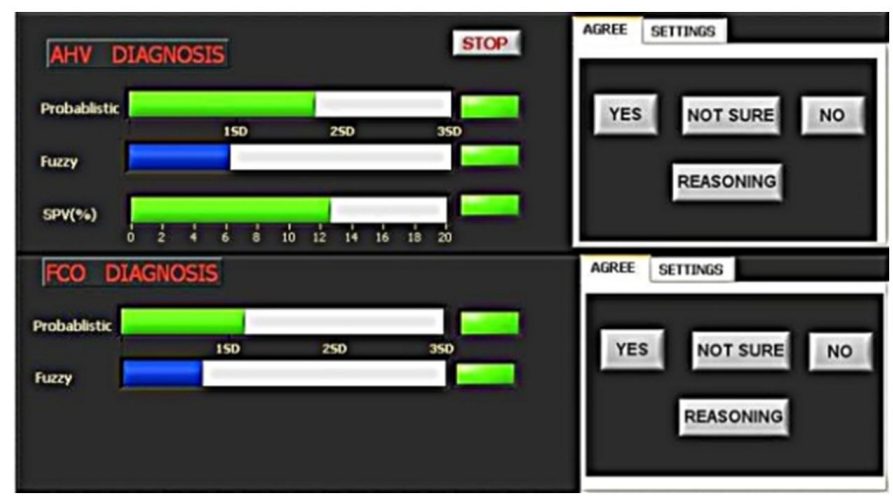

Figure 13. RT-SAAM screen with windows diagnoses. AHW (acute hypovolemia), hypovolemia; fall in cardiac output (FCO) [56]. 
With the implementation of a Multi-Modal Alarms System (MMAS) [58], RT-SAAM was able to diagnose also sympathetic activity, relative hypovolemia and inadequate anesthesia; diagnostic messages and alerts were sent every 10 seconds to MMAS. Every outcome alarm was connected to a specific sound that was directly transmitted to the anesthetist through a bluetooth headset. The MMAS display had two different modalities of presentation, depending on the presence or not of the symptoms (Figure 14).

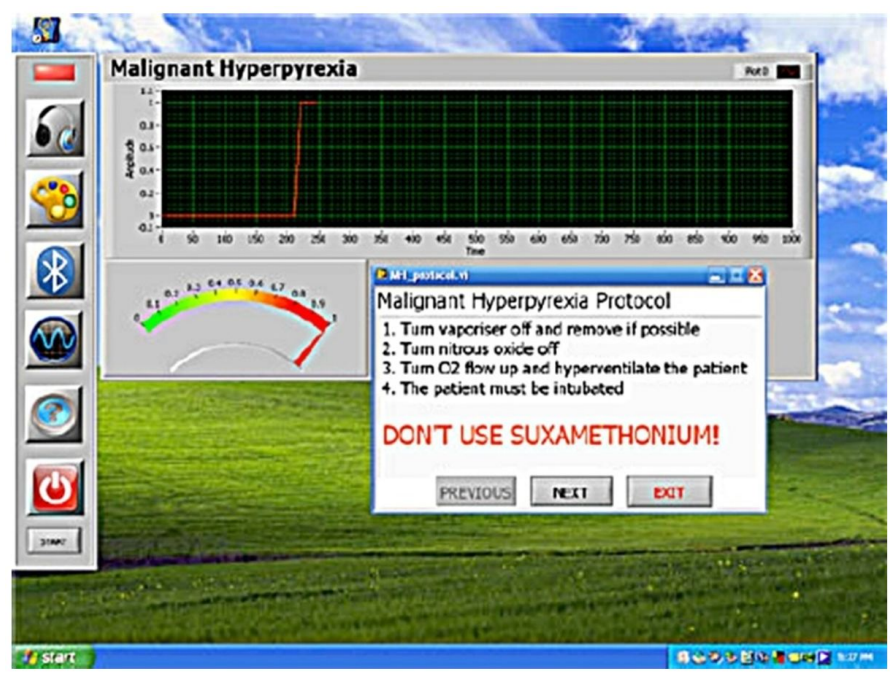

Figure 14. Alert modality of presentation [59].

In 2008, Perkin and Leaning presented Navigator, a DSS involved in the therapeutic control of the circulation and the oxygen delivery optimization and management [60]. They developed a mathematical model to create an algorithm for the control of circulation based on the values of the effective circulating volume (Pms), systemic vascular resistance (SVR) and heart performance (Eh). Using mathematical techniques, the values were derived from measured circulatory variables, the mean arterial pressure (MAP), the right atrial pressure (RAP) and the cardiac output $(\mathrm{CO})$ : corrected with a factor, $c$, that correlates with height, weight and age of the subject.

Through the combination of these values, Navigator supports the decision process with continuous therapeutic informations about the hemodynamic status and the oxygen delivery index related to the cardiac output, based on the entered hemoglobin and the arterial oxygen saturation (Spo2). The system display (Figure 15) is organized as such: on the right side, there is the current status of the patient, with his current values acquired from the monitors, target values and other data; on the left side, there is the patient's position (the red dot in the yellow arrow) on an orthogonal graph, in which: $x$-axis is the resistance axis (SVR values); $y$-axis is the volumetric axis (Pms values); MAP and CO are shown as lines corresponding to their upper 
and lower target ranges; the equivalent delivery oxygen indices are shown on the CO lines; the heart performance (Eh values) is displayed like a vertical axis parallel to the Pms axis.
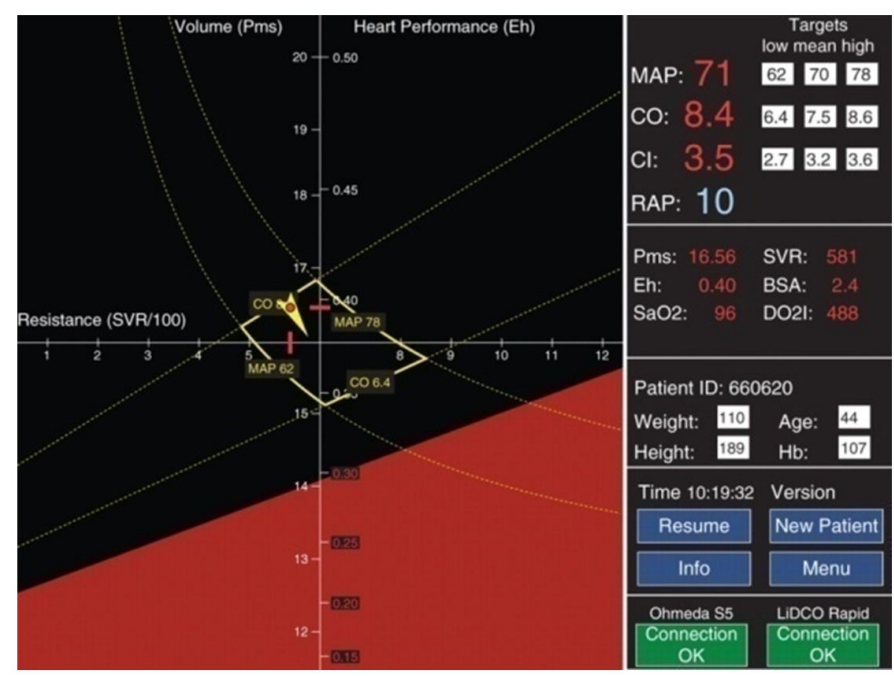

Figure 15. Navigator display [61].

\begin{tabular}{llll} 
& Guidance (n=57) & Control (n=48) & $P$ value \\
\hline ASD (mean standard distance from the central point of the target values) & & & \\
Entire period the patient was connected to Navigator & 1.71 (SD 0.65) & $1.92(\mathrm{SD} 0.98)$ & 0.202 \\
1-3 hours (SD) & $1.77(0.87)$ & $1.88(0.90)$ & 0.541 \\
3-6 hours (SD) & $1.62(0.83)$ & $1.96(1.38)$ & 0.123 \\
6-9 hours (SD) & $1.65(0.81)$ & $1.79(0.94)$ & 0.434 \\
9-12 hours (SD) & $1.57(0.73)$ & $1.84(0.96)$ & 0.124 \\
12-15 hours (SD) & $1.58(0.78)$ & $1.80(1.01)$ & 0.216 \\
15-18 hours (SD) & $1.76(0.80)$ & $1.93(0.94)$ & 0.388 \\
18-21 hours (SD) & $1.67(0.78)$ & $1.88(0.94)$ & 0.318 \\
21-24 hours (SD) & $1.92(0.96)$ & $1.73(0.97)$ & 0.563 \\
Average deviation in MAP to the central point of the target MAP range, mmHg & 7.3 (SD 2.68) & 7.4 (SD 2.14) & 0.976 \\
Average deviation in CO to the central point of the target CO range, 1/min & 0.99 (SD 0.47) & 1.05 (SD 0.69) & 0.566 \\
Mean percentage of time in the target cardiovascular range & 38.38 (SD 17.59) & 32.40 (SD 21.04) & 0.098 \\
Percentage of patients with clinically significant AF during connection & 5.08 & 5.77 & 1.00 \\
Percentage of patients with clinically significant AF during and after connection & 37.29 & 42.31 & 0.590 \\
Mean day 1 SOFA score & 5.89 (SD 2.88) & 6.50 (SD 3.36) & 0.335 \\
Mean day 2 SOFA score & 5.89 (SD 2.88) & 6.75 (SD 2.67) & 0.240 \\
Mean day 3 SOFA score & 6.00 (SD 1.63) & 9.20 (SD 1.92) & 0.011 \\
\hline
\end{tabular}

Table 2. $A S D=$ average standardized distance, $M A P=$ mean arterial pressure, $C O=$ cardiac output, $A F=$ atrial fibrillation, SOFA=Sequential Organ Failure Assessment [62]. 
Pellegrino et al [62] assessed Navigator in postoperative cardiac surgical patients. Fifty-seven patients received DSS-guided care and were compared with 48 patients who received conventional care. The performance of the system, considered as "average standardized distance" (ASD) between actual and target values of MAP and CO, was statistically not inferior to the control, and there were no significantly differences in the hospital length of stay (Table 2).

Sondergaard et al. [61] tested the Navigator's hemodynamic control and oxygen delivery during elective major abdominal surgery. They compared two groups of patients, one treated using DSS and the other one treated by expert anesthetists. They found a high concordance between the advices of the system and the intervention of the anesthetists.

Another complex DSS conceived to assist the anesthetist during surgery is Diagnesia [63]. It uses the input from the anesthesia panel to estimate the likelihood or unlikelihood of a diagnosis; it then gives the five most probable diagnoses in descending order (from the most to the least likely) with respective information that support or are against the evidence (Figure 16). Tested in 12 realistic situations from simulated anesthesia monitoring displays, its diagnoses were compared with those of a group of anesthesiologists, and in 11 test cases (92\%), the most probable diagnosis was the same; however, the system couldn't distinguish between two or more specific problems from the same category and couldn't deal with diagnosis in which the indicators were only observable but not measurable.

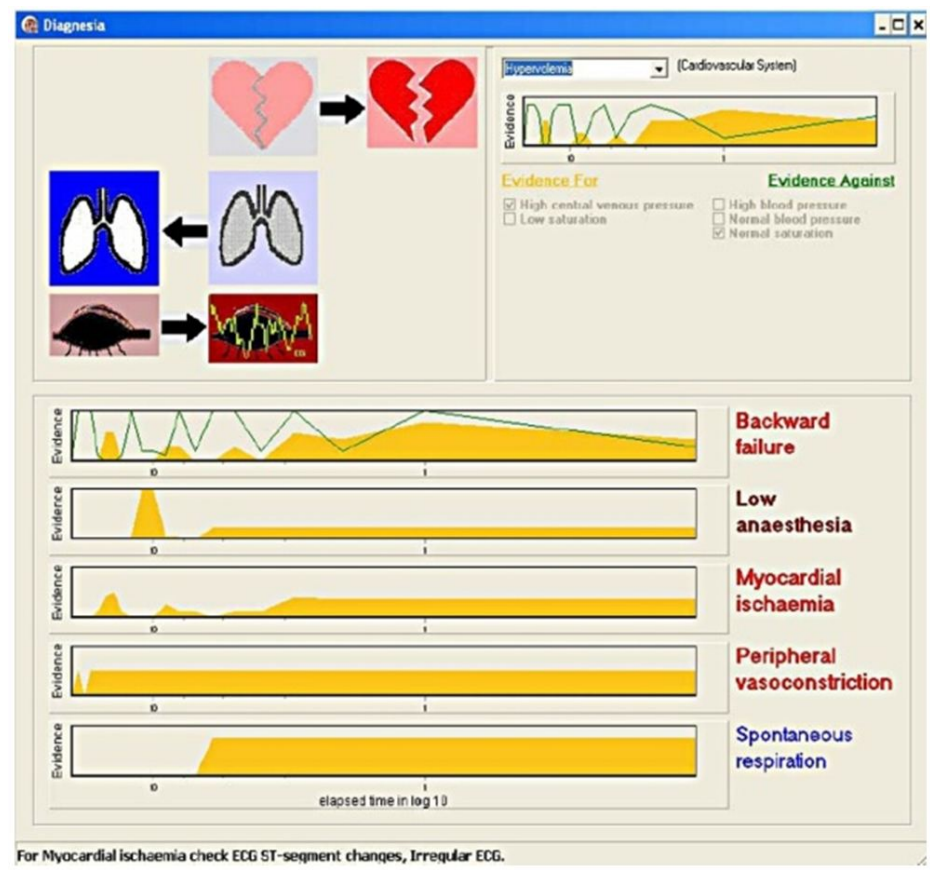

Figure 16. Graphical user interface [64]. 
Lastly, a hybrid system for conscious sedation (HSS) with DSS, was presented [65]. This system integrates closed loop sedation with a DSS, offering pop-up menus as smart alarms with several treatment advices for hemodynamic or respiratory adverse events, which need to be confirmed by the anesthetic team by clicking respective touch buttons on a touch screen (Figure 17).

Tested on two groups of 50 patients, the detection of critical events was significantly improved by the DSS, as shown in Table 3.

\begin{tabular}{|c|c|c|c|}
\hline & $\begin{array}{l}\text { Protocol Group } \\
(\mathrm{N}=50)\end{array}$ & $\begin{array}{l}\text { Control Group } \\
(\mathrm{N}=50)\end{array}$ & $\mathrm{P}$ \\
\hline Events not detected (\%) & 0 & 25 & $\begin{array}{l}\star<0.0001 \\
\#<0.000\end{array}$ \\
\hline AVG Delay (s) & $7.4 \pm 4.0$ & $32.6 \pm 21.7$ & 1 \\
\hline Critical events/h & $5.6 \pm 4.1$ & $7.4 \pm 5.1$ & $\# 0.05$ \\
\hline $\mathrm{MAP} / \mathrm{h}$ & $3.2 \pm 3.1$ & $3.7 \pm 3.6$ & N.S. \\
\hline $\mathrm{RR} / \mathrm{h}$ & $4.3 \pm 3.6$ & $3.8 \pm 4.1$ & N.S. \\
\hline $\mathrm{HR} / \mathrm{h}$ & $0.9 \pm 0.5$ & $0.7 \pm 0.3$ & N.S. \\
\hline Sat/h & $1.6 \pm 1.1$ & $2.2 \pm 2.4$ & N.S. \\
\hline
\end{tabular}

Table 3 Comparison of detecting critical events by the time [65].

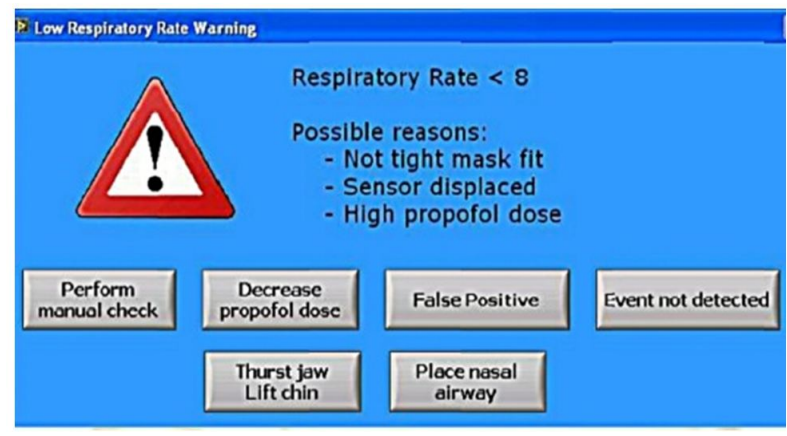

Figure 17. Pop-up menu for respiratory critical event [65].

\section{Decision Support Systems in Emergency Medicine}

Emergency medicine is one of the most difficult challenges for physicians. Diagnostic and therapeutic choices must be quick, immediate, even if there could be a significant inadequacy of information. Medical staff has to deal with many types of stressful situations: in-hospital emergency departments are often overcrowded [66-68], out-of-hospital emergency situations sometimes carry possible environmental risks. It is not possible to refuse care to anyone and there is also a high legal risk. All these elements can yield a huge stress load for 
the whole health care team [69-72]. These considerations lead to a request for decision support. There are several difficulties for designing systems for this environment and testing them. In a study of 2003, the diagnostic performance of two DSSs planned for an emergency department [73] were compared with expert decision making. Only in one third of the cases, the experts' diagnosis was within the top five diagnosis generated by the software.

We discuss further DSSs for in-hospital emergency medicine and DSSs for pre-hospital and out-of-hospital emergency medicine.

\subsection{Decision support systems for in-hospital emergency medicine}

Chest pain and abdominal pain represent principal causes of admission to the emergency room [74]. An example of a DSS for in-hospital emergencies is an artificial neural network designed to diagnose acute myocardial infarction [75]. It was tested in 118 patients, with a sensitivity of $92 \%$ and specificity of $96 \%$; without the input of the electrocardiogram, the sensitivity decreased to $86 \%$, while the specificity was $92 \%$. In a later study, the same DSS was tested in 331 patients with anterior chest pain in emergency department [76]. Whereas sensitivity and specificity of physicians in diagnosing myocardial infarction was $77.7 \%$ and $84.7 \%$, the DSS values were higher at $97.2 \%$ and $96.2 \%$, respectively. This suggests that the system performed better than physicians.

A simple DSS that could be useful in emergency medicine is an artificial neural network designed to detect microembolic Doppler signals [77]; it resulted in a specificity of $56.7 \%$ and sensitivity of $73.4 \%$, increasing to $75.9 \%$ in patients with mechanical prosthetic cardiac valves. However, this study did not test this system in emergency situations. Results about a favorable use of DSS to diagnose pulmonary embolism in emergency medicine in fact are not uniform. For example, the study of Roy et al. tried to evaluate the importance of the introduction of a computer-handled DSS in diagnosing pulmonary embolism compared with the use of paper guidelines [78]. The software yields a list of tests, specifying which of them is appropriate or inappropriate considering the pre-test probability, entered before by the physician according to the revised Geneva score; the DSS recommends as first choice the least invasive investigation among the appropriate tests. The system was compared with paper guidelines in two groups of patients, one with DSS and one without it. In the intervention group (the DSS group), there was an increase of appropriate diagnostic testing by $30.2 \%$ while in the other group it only increased by $10.9 \%$. The DSS was used in $80 \%$ of cases during real time intervention, suggesting a good performance in the emergency environment and good acceptance by physicians. A study by Drescher evaluated the impact of the integration of a computerized DSS in a computerized physician order entry on the frequency of positive CT angiography results for pulmonary embolism and the staff's acceptability of such a DSS[79]. The DSS was designed based on a modified Wells score to give diagnostic options to physicians when ordering a CT angiography or D-dimer testing (Figure 18). Although the study showed a superior performance of the DSS rather than physicians in ordering diagnostic investigations, adherence and acceptability of the DSS were quite low. Probably, the principle reasons were the time needed to enter data and mistrust in the effectiveness of the software. 


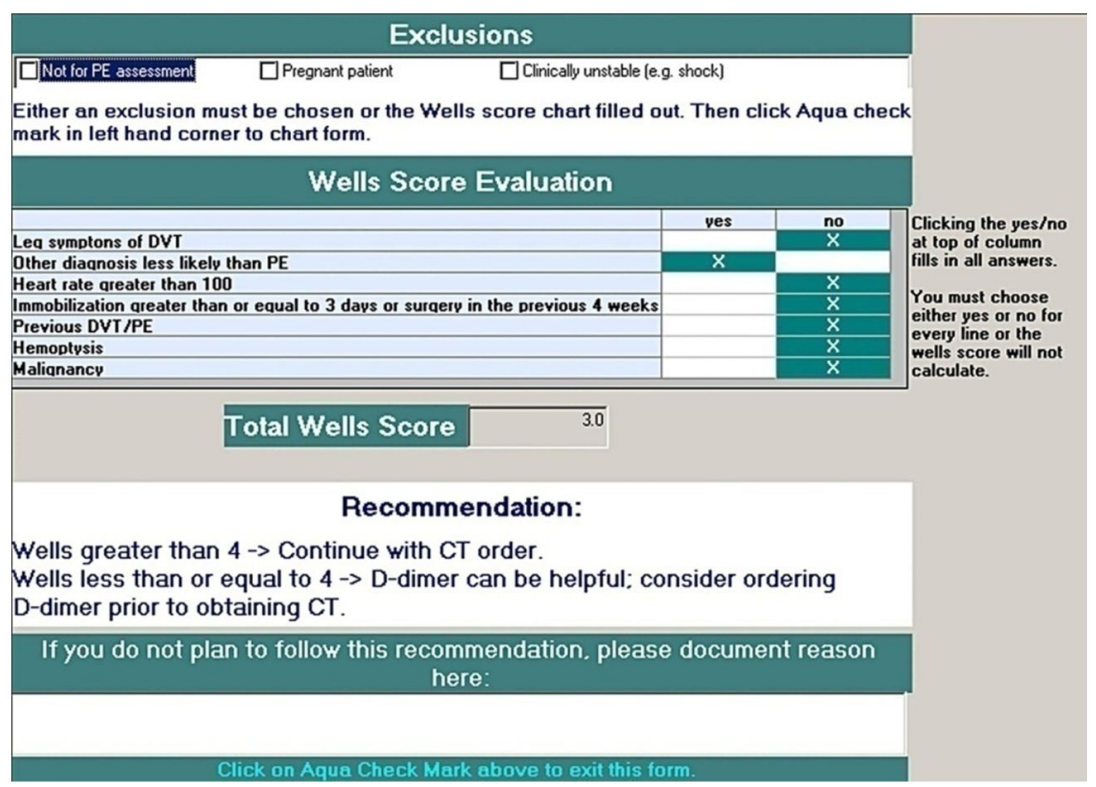

Figure 18. User interface for entering Wells criteria [79].

\subsection{Decision support systems for out-of-hospital and pre-hospital emergency medicine}

Decision support systems conceived for out-of hospital and pre-hospital interventions are complex systems designed for receiving and sending a multitude of inputs through a variety of platforms and different situations. These systems can interface with different signals, managing a great number of data also at distance. In his review [80], Nangalia describes five basic components of a telemedicine system: the acquisition of data through appropriate sensors; transmission of data from patient to clinician; ability to combine all data of different sources; decision support for appropriate action and response; storage of data.

One DSS used for pre-hospital emergency care is a system installed in ambulances that provides data communication, documentation, triaging and presentation of a checklist [81].

A similar pre-hospital DSS is iRevive, a system which permits rapid acquisition of data in an electronic format [82] through several components: vital sign sensors, wireless patient location and a central command center for discussing and collection of data, all linked by a network of wireless and hand-held computers. There is a combination of real-time sensor data, procedural data, and geographic data giving real-time decision support at three different hierarchical levels: at the local site, at the local command center and at the central command center, which is responsible for general coordination (Figure 19).

Another DSS for pre-hospital emergency care delivers real-time decision support during transport of trauma casualties [83]. It consists of a monitor that acquires data from patients 
in real-time, and a computer, that is implemented with three different types of software: the Controller software which records the data from monitor; the Shell software, which protects data by possible corruption and passes to the other software, and the Analysis software, which enables decision support. One important aim of this DSS is avoiding the loss or damage of information during the transport; this is possible by a special way of transmission of data that allows the system to know which data are not valid or missed.

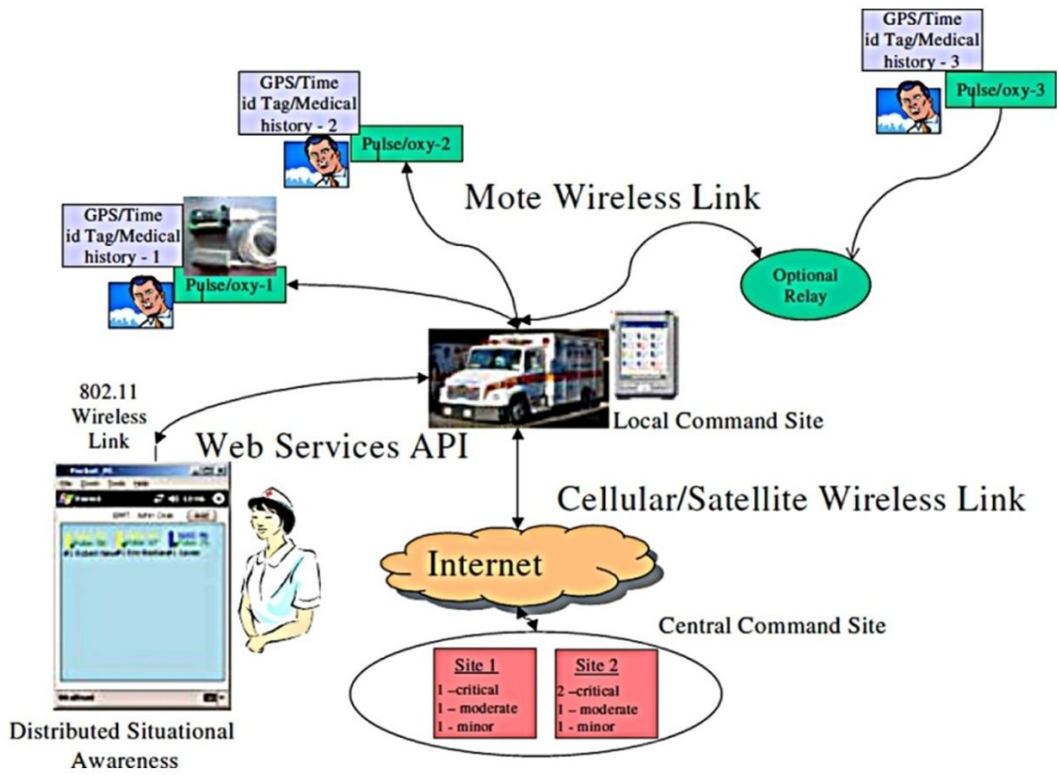

Figure 19. Basic architecture of iRevive [82].

Evaluated during a simulation of possible fault scenarios, the performance of each component was good and sufficient to prevent data corruption; decision support was considered useful.

\section{Decision Support Systems for Intensive Care Unit}

Intensive care medicine represents an equally challenging field for physicians. Patients are usually affected by multiple disease and need to be constantly supervised; often in a state of unconsciousness, patients cannot directly communicate with the practitioner and have to be monitored using a multitude of parameters, producing a significant amount of data. There are significant costs involved.

We will focus on general DSSs, DSSs for artificial ventilation and DSSs for infections. 


\subsection{General DSSs for ICU}

One DSS designed to deal with general issues of intensive care medicine is ACUDES (Architecture for Intensive Care Unit Decision Support), which takes into account the evolution of the patient following her/his diseases over time and gives information about illness and concomitant signs [84]. Another general DSS for intensive care unit is RHEA [85]; like ACUDES, it collects data from patients and gets information about adverse events and nosocomial infection risk for each patient. Data are entered manually; the system launches useful messages or alerts about the therapy by prediction models.

A general DSS designed for neuro-intensive care unit is iSyNCC (intelligent System for Neuro-Critical-Care) [86]. This system collects data from patients in a continuous way and uses them to provide decision support in terms of alerts or therapeutic messages, predicting also the patients' recovery. This is possible by the integration of four modules: data acquisition module, data storage module, data transmission module and user interface (Figure 20).

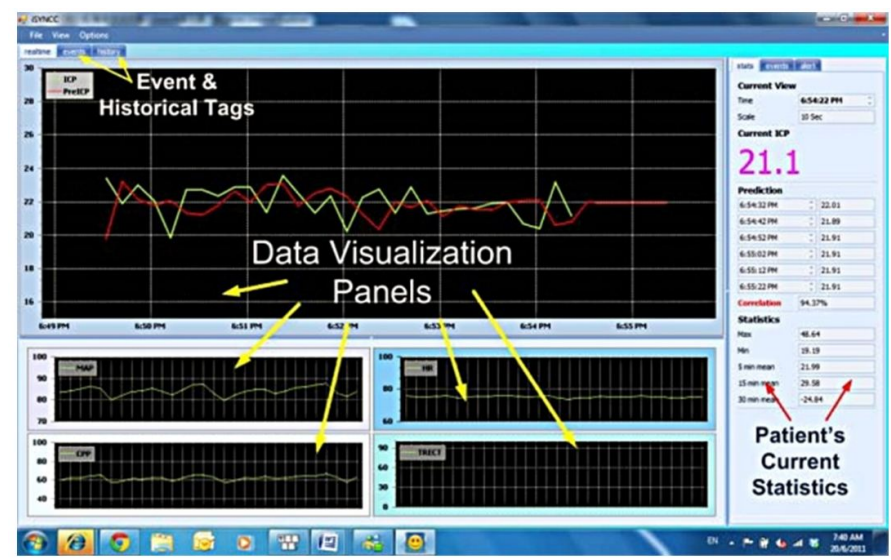

Figure 20. Graphical user interface [86].

\subsection{Decision support systems for artificial ventilation}

Systems for artificial ventilation have to be effective, safe and easy to use at the patient bedside. To be useful, its software must remove all noise and artifacts, processing only validate data. Ventilation requires constant monitoring, in order to ensure a timely weaning. Tehrani [87] suggests four possible barriers:

1. lack of accessibility,

2. no immunity to noise and erroneous data,

3. inadequate training for use of the systems, and

4. lack of implementation in commercial ventilators. 
The acceptability of DSSs by medical staff in the ICU is quite good; in his review [88], East found that DSSs were well accepted by ICU physicians and provided good performance improving outcome.

A DSS for artificial ventilation and weaning is the knowledge-based closed-loop system SmartCare ${ }^{\mathrm{TM}}$ [89]. Based on respiratory rate, tidal volume and end-tidal $\mathrm{CO}_{2}$, it takes a picture of the patient's present state, and using this picture, it continuously adapts the level of pressure support to maintain the patient in a respiratory comfort zone. The system contains a weaning protocol that gradually decreases the level of pressure support when patient's respiratory status improves (Figure 21). The use of the DSS resulted in more efficient weaning, with a decrease of the total duration of artificial ventilation from 12 to 7.5 days [90].

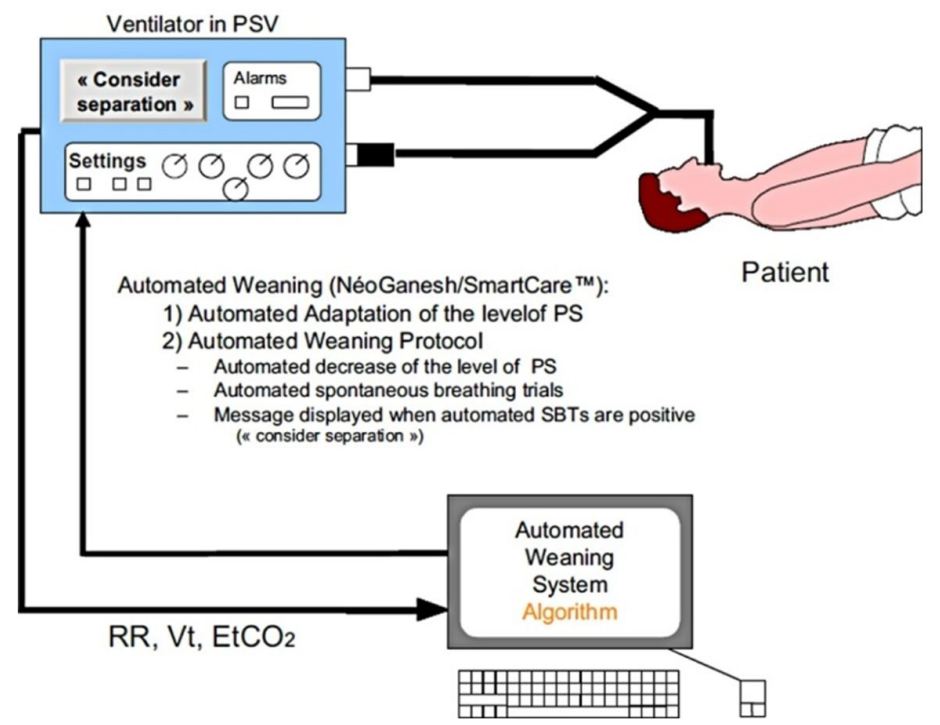

Figure 21. Working principles of NeoGanesh/SmartCare. PS, pressure support; SBT, spontaneous breathing trial [91].

Another closed-loop ventilation system is IntelliVent-ASV. The system provides automatic setting of ventilator parameters and closed-loop regulation based on the inputs of ET$\mathrm{CO}_{2}, \mathrm{SpO}_{2}$ and $\mathrm{FiO}_{2}$ after individualization of correct target ranges. Initial results appear interesting [92].

A hybrid knowledge-based and physiological model-based DSS is the Sheffield Intelligent Ventilator Advisor (SIVA). It gives advices and adaptive patient-specific decision support using $\mathrm{FiO}_{2}$, PEEP, inspiratory pressure and ventilatory rate. When compared with expert decision making, appropriated decision making was obtained with the system and the control of blood gases was similar in both groups [93].

Another DSS for artificial ventilation is the INVENT project, based on physiological modelling. This system uses two software modules to process patient data to suggest specific ven- 
tilator settings. These modules are the Automatic Lung Parameters Estimator (ALPE system), that is based on a model of pulmonary gas exchange with particular attention to the oxygen transport, and the system for the arterializations of venous blood (ARTY system), integrating arterial values from venous blood. All data produced are used to send suggestions of ventilator settings based on the prediction of outcome (Figure 22).

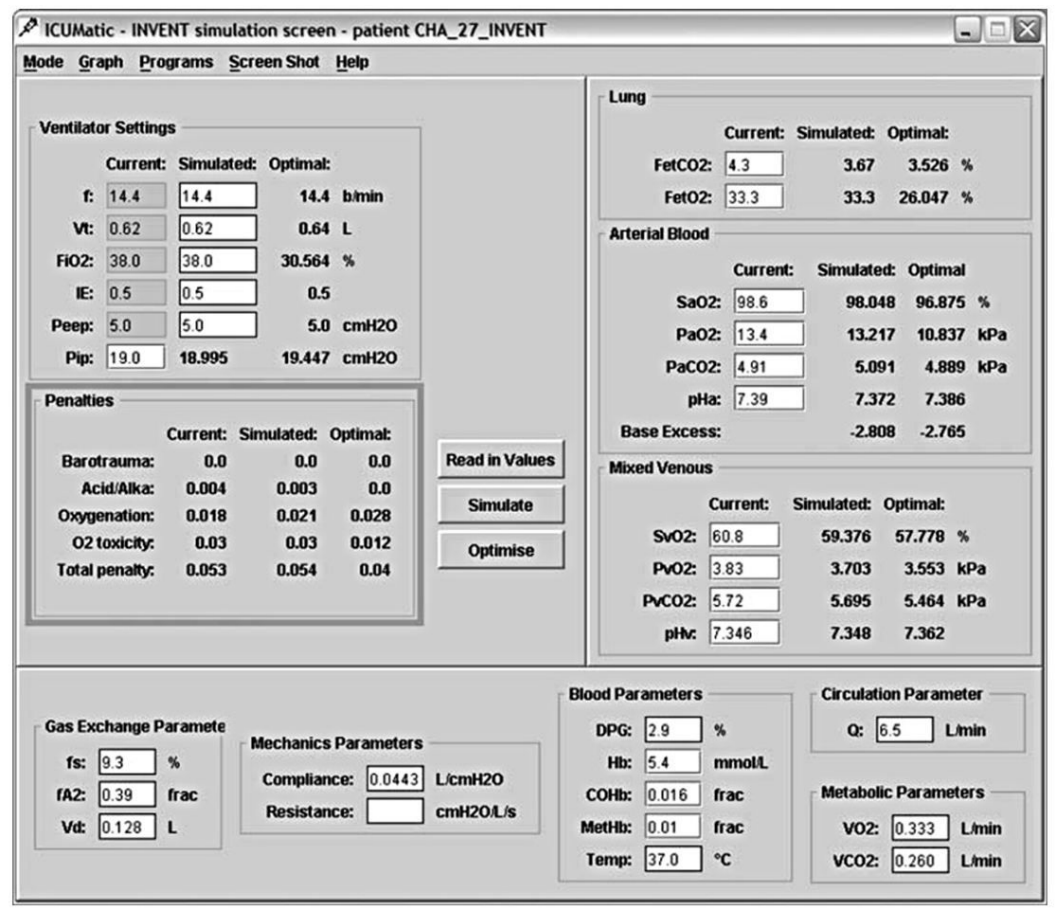

Figure 22. Graphical user interface with its 3 sections. The left side shows the ventilator settings and penalties, displayed as current, simulated and optimal. The right side displays variables of lungs, arterial and venous blood described as current, simulated and optimal value. On the bottom, patient specific parameters and related organ systems. In this illustration, data of a single post-operative cardiac patient are showed [94].

\subsection{Decision support systems for infections in ICU}

Infections in ICU represent $26 \%$ of nosocomial infections [95] and usually are accompanied by a high rate of serious problems like sepsis and mortality. Bacteria in the ICU are more resistant to antibiotic therapy. Patients in ICU are monitored by a variety of invasive devices; these devices can transmit germs directly. The most frequent nosocomial infections are catheter-associated urinary infections, and occur in about 35\% of the cases, characterized by low mortality and costs, while bloodstream infections and respiratory ventilator-associated infections occur at about $15 \%$ of the cases, but are associated with high mortality and costs $[34,95]$. For all these reasons, antimicrobial infection surveillance in ICU has to be very strict, usually more than in other hospital wards [95]. 
After observation of infection management in a 21-bed mixed medical/surgical adult ICU, an Australian group [96] designed a software, ADVISE, that allowed the digitalization of patient data to create appropriate antibiotic recommendations in real-time. Messages are not only strictly about therapy, but also about the general theory of the specific pathogens or other patient-related information (Figure 23). Comparison between 6 months before and 6 months after the use of this DSS revealed a better rationalization of the antibiotic use, with a reduction of $10.5 \%$ in the overall prescription of antiobiotics, mostly cephalosporin and vancomycin [97].
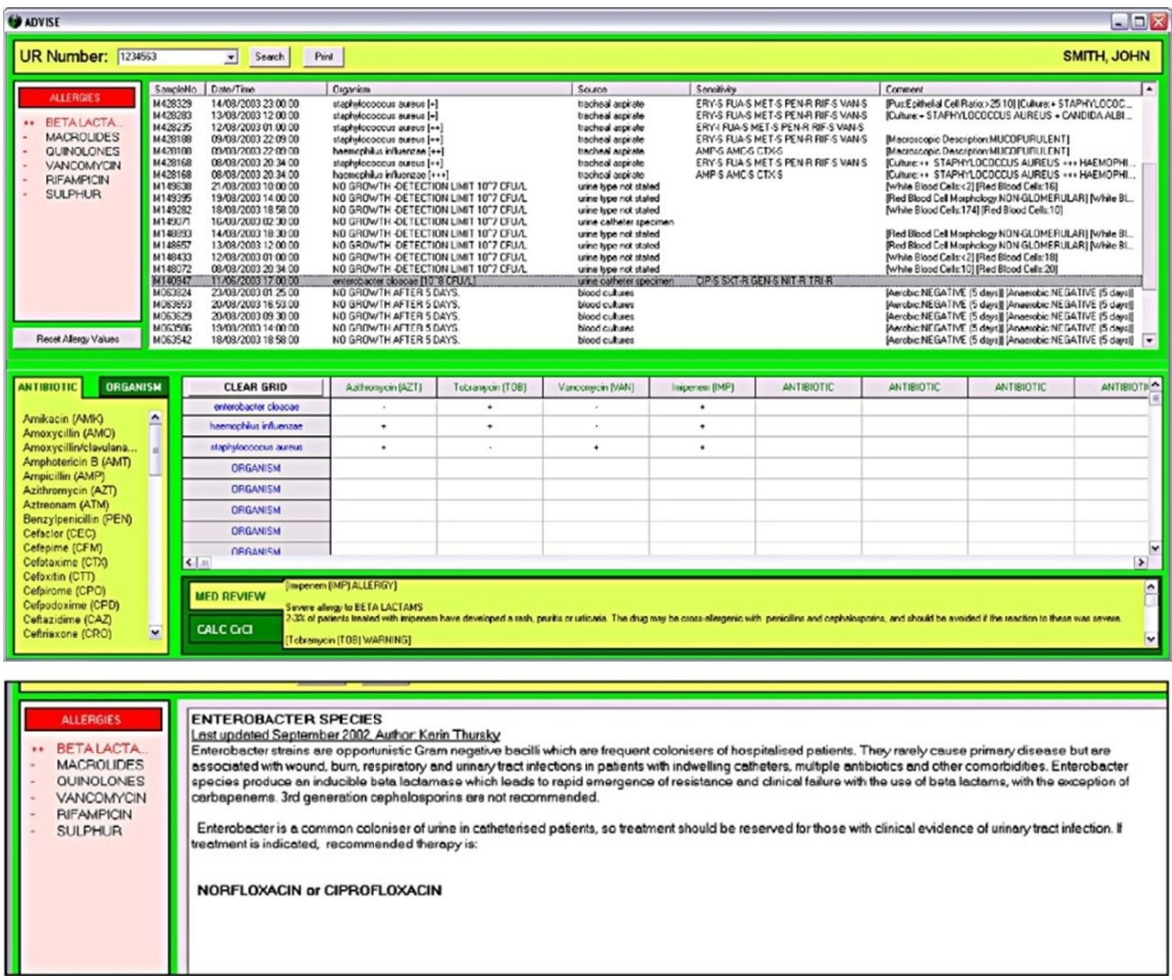

Figure 23. Screenshot of ADVISE showing with microbiology results, allergy profile, and antimicrobial/isolate matrix. It is displayed the medical review panel, alerting the user to potential allergy risks or overlapping antibiotic coverage, and the rule-based recommendation generated by clicking on the isolate in the tabular view [96].

A sepsis computer protocol was implemented in a 27-bed surgical ICU to manage sepsis [98]. International validated scores were used to make a daily assessment of outcome together with all patient data. All this material was placed in a computer protocol, with nine logical diagrams displayed individually to be used at bedside. Computer instructions had to be confirmed by the clinician before the execution of interventions. The system's performance was evaluated via the comparison with paper guidelines: the DSS improved the administration of antibiotics, supported therapeutic decision making and reduced mortality. 
The COSARA research project (Computer-based Surveillance and Alerting of nosocomial infections, Antimicrobial Resistance and Antibiotic consumption in ICU) is a complex system that automatically acquires patient data and records all information about the patient's clinical history, therapy and antibiotic resistance, providing decision support through visual presentation of graphs, icons, visual bars, pop-ups and audible alerts [99]. The DSS consists of different modules, having each one the ability to manage some functions, as e.g. X-ray photos. For each antibiotic prescription, there is a pop-up menu prompting the physician to register the motivation for starting therapy (Figure 24). Clinical decision support is delivered through mail or messages based on guidelines. After 3 months of implementation, clinical outcome was improved.

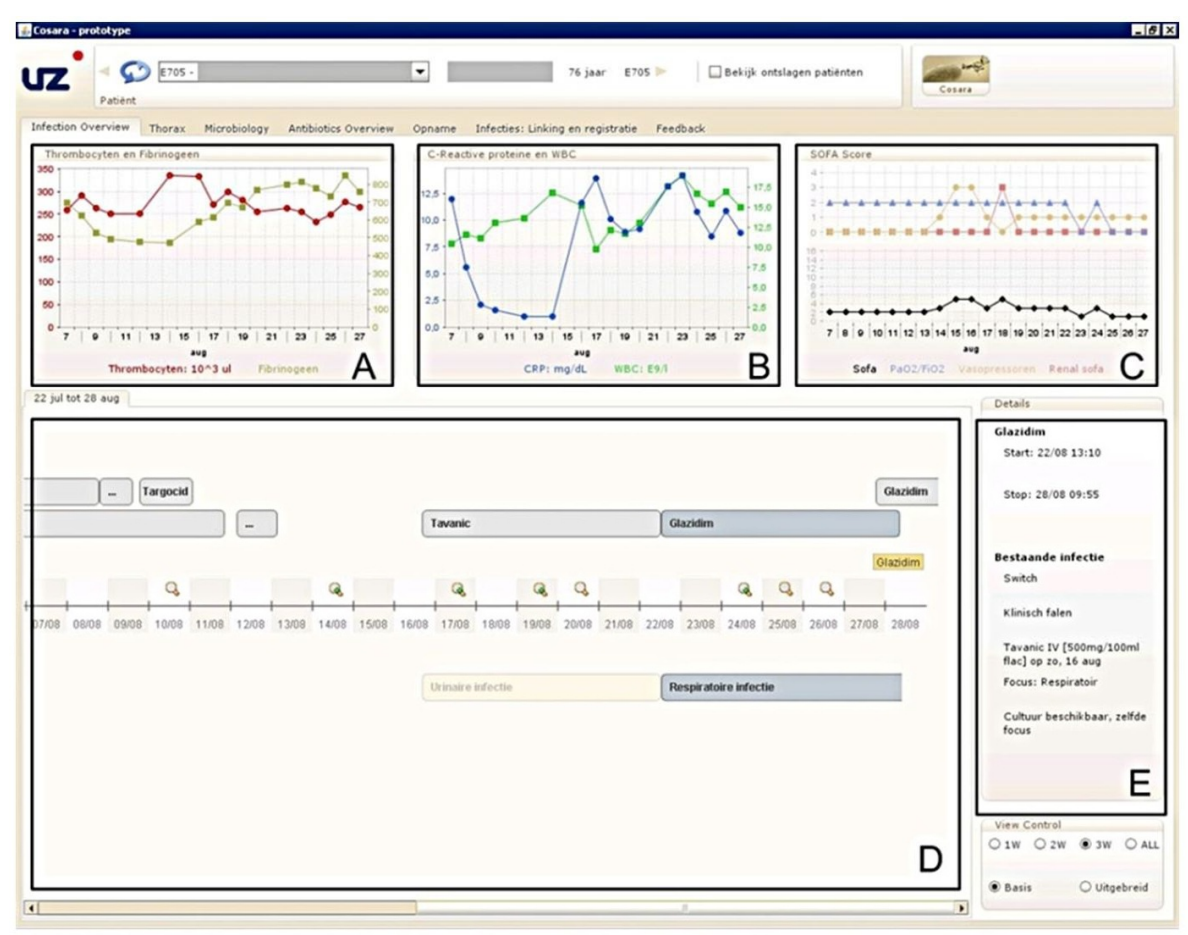

Figure 24. Screenshot with infections and antibiotics history; there also graphs that represent common used values [99].

\section{Discussion}

Decision support systems have been successfully developed in the areas of intensive care, emergency medicine and anesthesia. These are areas with an overload of information and ne- 
cessity to react quickly and adjust the reactions throughout a short time interval. More and more, DSSs are integrated in AIMS in the perioperative period. These have been successfully used to configure checklists to reduce the incidence of intraoperative problems, thus even reducing costs significantly. Decision support systems in that context can reduce the amount of laboratory investigations performed. More anesthetic specific DSSs are also being developed, allowing risk stratification for difficult intubations, better PONV prophylaxis, appropriate timing of antibiotic prophylaxis and thus reducing the incidence of wound infections. In the arena of emergency medicine, both in- and out-of-hospital DSSs have been created and successfully tested. They range from systems to help identify microemboli or pulmonary embolism, to systems to help coordinating the pre-hospital logistics. Some of these systems are met with skepticism by physicians because they fear of handing over decision making to these systems. In ICU units, DSSs can help with the setup of artificial ventilation and significantly reduce infections: this is specifically important in the complex decision making and establishment of appropriate treatment options in patients suffering from sepsis.

\section{Conclusion}

Decision support systems are useful tools in modern medicine. They can improve clinical practice, adherence to best evidence based medicine and, in some cases, clinical and health education and patients outcomes.

In the operating room, DSSs can be effective to deal with a variety of problems. In emergency medicine, they have shown good performance, but their acceptability by clinicians has not been sufficient. Decision support systems in intensive care medicine allow the possibility to manage a large number of data whilst allowing the clinician to follow them in a more efficient way. However, a widespread use of these systems is obstructed by economical and cultural barriers; a greater involvement of the medical users in the design of the systems, including better user interfaces, could improve their clinical acceptance. DSSs are software designed to assist the physician but are not a substitutes of this figure.

Key messages

1. Decision support systems improve quality of practice and patient safety.

2. There is an increased development of DSSs in any medical field, specifically in the area of critical care medicine.

3. Despite the increasing request, cultural and economical barriers obstruct the diffusion of these systems. 


\section{Author details}

Thomas M. Hemmerling ${ }^{1 *}$, Fabrizio Cirillo ${ }^{3}$ and Shantale Cyr ${ }^{2}$

*Address all correspondence to: thomas.hemmerling@mcgill.ca

1 Dept. of Anesthesia, McGill University \& Institute of Biomedical Engineering, University of Montreal, Montreal, Canada

2 Dept. of Anesthesia, McGill University

3 Dept. of Anesthesia, University of Naples, Italy

\section{References}

[1] Hemmerling, T. M. (2011). Decision Support Systems in Anesthesia, Emergency Medicine and Intensive Care Medicine, in Practice and Challenges in Biomedical Related Domain, J.S. C., Editor, InTech, 239-260.

[2] Bates, D. W., \& Gawande, A. A. (2003). Improving Safety with Information Technology. New England Journal of Medicine, 348(25), 2526-2534.

[3] Miller, R. A. (1994). Medical diagnostic decision support systems--past, present, and future: a threaded bibliography and brief commentary. J Am Med Inform Assoc, 1(1), $8-27$.

[4] de Dombal, F. T., Hartley, J. R., \& Sleeman, D. H. (1969). A computer-assisted system for learning clinical diagnosis. Lancet, 145-148.

[5] Peleg, M., \& Tu, S. (2006). Decision support, knowledge representation and management in medicine. Yearb Med Inform, 72-80.

[6] Dunsmuir, D., et al. (2008). A knowledge authoring tool for clinical decision support. J Clin Monit Comput, 22(3), 189-198.

[7] Hawamdeh, Z. M., et al. (2012). Development of a decision support system to predict physicians' rehabilitation protocols for patients with knee osteoarthritis. Int J Rehabil Res.

[8] Su, C. T., et al. (2012). Prediagnosis of Obstructive Sleep Apnea via Multiclass MTS. Comput Math Methods Med, 212498.

[9] Shelton, R. C., \& Trivedi, M. H. (2011). Using algorithms and computerized decision support systems to treat major depression. J Clin Psychiatry, 72(12), e36.

[10] Ebell, M. H., et al. (2012). Development and validation of a clinical decision rule for the diagnosis of influenza. J Am Board Fam Med, 25(1), 55-62. 
[11] Legros, B., et al. (2012). Development of an electronic decision tool to support appropriate treatment choice in adult patients with epilepsy--Epi-Scope((R)). Seizure, 21(1), 32-39.

[12] Van Belle, V. M., et al. (2012). A mathematical model for interpretable clinical decision support with applications in gynecology. PLoS One, 7(3), e34312.

[13] Garg, A. X., et al. (2005). Effects of computerized clinical decision support systems on practitioner performance and patient outcomes: a systematic review. JAMA, 293(10), 1223-1238.

[14] Kawamoto, K., et al. (2005). Improving clinical practice using clinical decision support systems: a systematic review of trials to identify features critical to success. BMJ, 330(7494), 765.

[15] Pearson, S. A., et al. (2009). Do computerised clinical decision support systems for prescribing change practice? A systematic review of the literature (1990-2007). BMC Health Serv Res, 9, 154.

[16] Kooij, F. O., et al. (2008). Decision support increases guideline adherence for prescribing postoperative nausea and vomiting prophylaxis. Anesth Analg, 106(3), 893-898, table of contents.

[17] Hunt, D. L., et al. (1998). Effects of computer-based clinical decision support systems on physician performance and patient outcomes: a systematic review. JAMA, 280(15), 1339-1346.

[18] Bates, D. W., et al. (2001). Reducing the frequency of errors in medicine using information technology. J Am Med Inform Assoc, 8(4), 299-308.

[19] Glavin, R. J. (2009). Excellence in anesthesiology: the role of nontechnical skills. Anesthesiology, 110(2), 201-203.

[20] Seim, A. R., \& Sandberg, W. S. (2010). Shaping the operating room and perioperative systems of the future: innovating for improved competitiveness. Curr Opin Anaesthesiol, 23(6), 765-771.

[21] Hemmerling, T. M. (2009). Automated anesthesia. Curr Opin Anaesthesiol, 22(6), 757-763.

[22] Mort, M, et al. (2005). Safe asleep? Human-machine relations in medical practice. Soc Sci Med, 61(9), 2027-2037.

[23] Reason, J. (2005). Safety in the operating theatre- Part 2: human error and organisational failure. Qual Saf Health Care, 14(1), 56-60.

[24] Sawa, T., \& Ohno-Machado, L. (2001). Generation of dynamically configured check lists for intra-operative problems using a set of covering algorithms. Proceedings / AMIA... Annual Symposium, 593-597. 
[25] Finegan, B., et al. (2005). Selective ordering of preoperative investigations by anesthesiologists reduces the number and cost of tests. Canadian Journal of Anesthesia / Journal canadien d'anesthésie, 52(6), 575-580.

[26] Flamm, M., et al. (2011). Non-adherence to guidelines for preoperative testing in a secondary care hospital in Austria: the economic impact of unnecessary and double testing. Eur J Anaesthesiol, 28(12), 867-873.

[27] Tisavipat, S., \& Suesaowaluk, P. (2011). Development of an automated decision system for selection of preoperative investigations based on cartesian product and gaussian distribution, in Information Reuse and Integration (IRI). IEEE International Conference on, 502-503.

[28] Gannon, K. (1991). Mortality associated with anaesthesia. A case review study. Anaesthesia, 46(11), 962-966.

[29] Braz, L. G., et al. (2009). Mortality in anesthesia: a systematic review. Clinics (Sao Paulo, 64(10), 999-1006.

[30] Yan, Q., et al. (2009). SVM-based decision support system for clinic aided tracheal intubation predication with multiple features. Expert Systems with Applications, 6588-6592.

[31] Chau, A., \& Ehrenfeld, J. M. (2011). Using real-time clinical decision support to improve performance on perioperative quality and process measures. Anesthesiol Clin, 29(1), 57-69.

[32] Wanderer, J. P., Sandberg, W. S., \& Ehrenfeld, J. M. (2011). Real-time alerts and reminders using information systems. Anesthesiol Clin, 29(3), 389-396.

[33] Kooij, F. O., et al. (2012). Automated reminders decrease postoperative nausea and vomiting incidence in a general surgical population. Br J Anaesth.

[34] Burke, J. P. (2003). Infection control- a problem for patient safety. N Engl J Med, 348(7), 651-656.

[35] Leape, L. L., et al. (1991). The nature of adverse events in hospitalized patients Results of the Harvard Medical Practice Study II. N Engl J Med, 324(6), 377-384.

[36] Auerbach, A. D. (2001). Prevention of surgical site infections. Making Health Care Safer: A Critical Analysis of Patient Safety Practices, 221.

[37] Kirkland, K. B., et al. (1999). The impact of surgical-site infections in the 1990s: attributable mortality, excess length of hospitalization, and extra costs. Infect Control Hosp Epidemiol, 20(11), 725-730.

[38] Gyssens, I. C. (1999). Preventing postoperative infections: current treatment recommendations. Drugs, 57(2), 175-185. 
[39] Dellinger, E. P. (2007). Prophylactic antibiotics: administration and timing before operation are more important than administration after operation. Clin Infect Dis, 44(7), 928-930.

[40] O'Reilly, M., et al. (2006). An anesthesia information system designed to provide physician-specific feedback improves timely administration of prophylactic antibiotics. Anesth Analg, 103(4), 908-912.

[41] Nair, B. G., et al. (2010). Feedback mechanisms including real-time electronic alerts to achieve near $100 \%$ timely prophylactic antibiotic administration in surgical cases. Anesth Analg, 111(5), 1293-1300.

[42] Nair, B. G., et al. (2011). Automated electronic reminders to improve redosing of antibiotics during surgical cases: comparison of two approaches. Surg Infect (Larchmt), 12(1), 57-63.

[43] Gordon, S. M. (2006). Antibiotic prophylaxis against postoperative wound infections. Cleve Clin J Med, 73(1), S42-S45.

[44] Steinberg, J. P., et al. (2009). Timing of antimicrobial prophylaxis and the risk of surgical site infections: results from the Trial to Reduce Antimicrobial Prophylaxis Errors. Ann Surg, 250(1), 10-16.

[45] Eden, A., et al. (2009). The impact of an electronic reminder on the use of alarms after separation from cardiopulmonary bypass. Anesth Analg, 108(4), 1203-1208.

[46] Krol, M., \& Reich, D. L. (2000). Development of a decision support system to assist anesthesiologists in operating room. J Med Syst, 24(3), 141-146.

[47] Mashour, G. A., et al. (2009). A novel electronic algorithm for detecting potentially insufficient anesthesia: implications for the prevention of intraoperative awareness. $J$ Clin Monit Comput, 23(5), 273-277.

[48] Hooper, B., et al. (1997). A fuzzy logic based decision support system for low-flow closed-loop anaesthesia, in Fuzzy Systems. Proceedings of the Sixth IEEE International Conference on, 1615-1620, 3.

[49] Mirza, M., Gholamhosseini, H., \& Harrison, M. J. (2010). A fuzzy logic-based system for anaesthesia monitoring. Conf Proc IEEE Eng Med Biol Soc, 3974-3977.

[50] Baig, M. M., et al. (2011). Detection and classification of hypovolaemia during anaesthesia, in Engineering in Medicine and Biology Society, EMBC. Annual International Conference of the IEEE, 357-360, 1557-170X.

[51] Lowe, A. (1999). Evidential Inference for Fault Diagnosis, in Mechanical Engineering, University of Auckland, Auckland.

[52] Lowe, A., Harrison, M. J., \& Jones, R. W. (1999). Diagnostic monitoring in anaesthesia using fuzzy trend templates for matching temporal patterns. Artif Intell Med, 16(2), 183-199. 
[53] Rosenberg, H., et al. (2007). Malignant hyperthermia. Orphanet J Rare Dis, 2, 21.

[54] Lowe, A., \& Harrison, M. J. (1999). Computer-enhanced diagnosis of malignant hyperpyrexia. Anaesth Intensive Care, 27(1), 41-44.

[55] Lowe, A., Jones, R. W., \& Harrison, M. J. (2001). The graphical presentation of decision support information in an intelligent anaesthesia monitor. Artif Intell Med, 22(2), 173-191.

[56] Gohil, B., et al. (2007). Intelligent monitoring of critical pathological events during anesthesia. Conf Proc IEEE Eng Med Biol Soc, 4343-4346.

[57] Lai, H. Y., et al. (2004). Effect of esmolol on positive-pressure ventilation-induced variations of arterial pressure in anaesthetized humans. Clin Sci (Lond), 107(3), 303-308.

[58] Ken, Lee. K. J. T. Y., Plimmer, B., \& Harrison, M. (2008). Real-time Anaesthesia Diagnosis Display System with Multi-Modal Alarms.

[59] Lee, K., et al. (2008). Real-time anaesthesia diagnosis display system with multi-modal alarms. ACM.

[60] Parkin, W. G., \& Leaning, M. S. (2008). Therapeutic control of the circulation. J Clin Monit Comput, 22(6), 391-400.

[61] Sondergaard, S., et al. (2012). High concordance between expert anaesthetists' actions and advice of decision support system in achieving oxygen delivery targets in highrisk surgery patients. $\mathrm{Br}$ J Anaesth.

[62] Pellegrino, V. A., et al. (2011). Computer based haemodynamic guidance system is effective and safe in management of postoperative cardiac surgery patients. Anaesth Intensive Care, 39(2), 191-201.

[63] Kizito, J. (2008). Diagnesia: A Prototype of a Decision Support System for Anesthetists, in Broadband Communications, Information Technology \& Biomedical Applications. Third International Conference on, 12-19.

[64] Kizito, J. (2009). Decision Support in the Operating Theatre- Usability Aspects, in Strenghtening the Role of ICT in Development, J.M.L. Kizza, K., Nath, R., Aisbett, J., Vir, P., Editor, Fountain Publishers, Kampala, 109-117.

[65] Hemmerling, T. M., Arbeid, E., \& Tang, L. (2011). HSS'- A Novel Hybrid System for Conscious Sedation. in Annual Conference of Society of Technology in Anesthesia: Las Vegas, 34 .

[66] Mc Cabe, J. B. (2001). Emergency department overcrowding: a national crisis. Acad Med, 76(7), 672-674.

[67] Derlet, R. W., \& Richards, J. R. (2000). Overcrowding in the nation's emergency departments: Complex causes and disturbing effects. Ann Emerg Med, 35(1), 63-68. 
[68] Olshaker, J. S., \& Rathlev, N. K. (2006). Emergency Department overcrowding and ambulance diversion: the impact and potential solutions of extended boarding of admitted patients in the Emergency Department. J Emerg Med, 30(3), 351-356.

[69] Kuhn, G., Goldberg, R., \& Compton, S. (2009). Tolerance for uncertainty, burnout, and satisfaction with the career of emergency medicine. Ann Emerg Med, 54(1), 106-113 e6.

[70] Popa, F., et al. (2010). Occupational burnout levels in emergency medicine--a nationwide study and analysis. J Med Life, 3(3), 207-215.

[71] Healy, S., \& Tyrrell, M. (2011). Stress in emergency departments: experiences of nurses and doctors. Emerg Nurse, 19(4), 31-37.

[72] Schmitz, G. R., et al. (2012). Strategies for coping with stress in emergency medicine: Early education is vital. J Emerg Trauma Shock, 5(1), 64-69.

[73] Graber, M. A., \& Van Scoy, D. (2003). How well does decision support software perform in the emergency department? Emerg Med J, 20(5), 426-428.

[74] Niska, R., Bhuiya, F., \& Xu, J. (2007). National Hospital Ambulatory Medical Care Survey: emergency department summary. Natl Health Stat Report, 2010(26), 1-31.

[75] Baxt, W. G. (1990). Use of an Artificial Neural Network for Data Analysis in Clinical Decision-Making: The Diagnosis of Acute Coronary Occlusion. Neural Computation, 2(4), 480-489.

[76] Baxt, W. G. (1991). Use of an Artificial Neural Network for the Diagnosis of Myocardial Infarction. Ann Intern Med, 115(11), 843-848.

[77] Kemeny, V., et al. (1999). Automatic embolus detection by a neural network. Stroke, 30(4), 807-810.

[78] Roy, P. M., et al. (2009). A computerized handheld decision-support system to improve pulmonary embolism diagnosis: a randomized trial. Ann Intern Med, 151(10), 677-686.

[79] Drescher, F. S., et al. (2011). Effectiveness and acceptability of a computerized decision support system using modified Wells criteria for evaluation of suspected pulmonary embolism. Ann Emerg Med, 57(6), 613-621.

[80] Nangalia, V., Prytherch, D. R., \& Smith, G. B. (2010). Health technology assessment review: remote monitoring of vital signs--current status and future challenges. Crit Care, 14(5), 233.

[81] Karlsten, R., \& Sjoqvist, B. A. (2000). Telemedicine and decision support in emergency ambulances in Uppsala. J Telemed Telecare, 6(1), 1-7.

[82] Gaynor, M., et al. (2005). A dynamic, data-driven, decision support system for emergency medical services. Computational Science-ICCS, 61-100. 
[83] Khitrov, M. Y., et al. (2009). A platform for testing and comparing of real-time decision-support algorithms in mobile environments. Conference Proceedings. Annual International Conference of the IEEE Engineering in Medicine $\mathcal{E}$ Biology Society, 3417-3420.

[84] Palma, J., et al. (2002). ACUDES: architecture for intensive care units decision support. in Engineering in Medicine and Biology. 24th Annual Conference and the Annual Fall Meeting of the Biomedical Engineering Society EMBS/BMES Conference, Proceedings of the Second Joint.

[85] Metais, E., Nakache, D., \& Timsit, J. F. (2006). Rhea: A Decision Support System for Intensive Care Units. 4th International Multiconference on Computer Science and Information Technology CSIT, 5-7.

[86] Feng, M., et al. (2011). iSyNCC: an intelligent system for patient monitoring \& clinical decision support in Neuro-Critical-Care. Conf Proc IEEE Eng Med Biol Soc, 6426-6429.

[87] Tehrani, F. T., \& Roum, J. H. (2008). Intelligent decision support systems for mechanical ventilation. Artif Intell Med, 44(3), 171-182.

[88] East, T. D., et al. (1999). Efficacy of computerized decision support for mechanical ventilation: results of a prospective multi-center randomized trial. Proc AMIA Symp, 251-255.

[89] Dojat, M., et al. (1992). A knowledge-based system for assisted ventilation of patients in intensive care units. Int J Clin Monit Comput, 9(4), 239-250.

[90] Lellouche, F., et al. (2006). A multicenter randomized trial of computer-driven protocolized weaning from mechanical ventilation. Am J Respir Crit Care Med, 174(8), 894-900.

[91] Lellouche, F., \& Brochard, L. (2009). Advanced closed loops during mechanical ventilation (PAV, NAVA, ASV, SmartCare). Best Practice E Research Clinical Anaesthesiology, 23(1), 81-93.

[92] Arnal, J. M., et al. (2012). Safety and efficacy of a fully closed-loop control ventilation $($ IntelliVent-ASV $((\mathrm{R})))$ in sedated ICU patients with acute respiratory failure: a prospective randomized crossover study. Intensive Care Med, 38(5), 781-787.

[93] Kwok, H. F., et al. (2004). SIVA: a hybrid knowledge-and-model-based advisory system for intensive care ventilators. IEEE Trans Inf Technol Biomed, 8(2), 161-172.

[94] Rees, S. E., et al. (2006). Using physiological models and decision theory for selecting appropriate ventilator settings. J Clin Monit Comput, 20(6), 421-429.

[95] Wenzel, R. P., et al. (1983). Hospital-acquired infections in intensive care unit patients: an overview with emphasis on epidemics. Infect Control, 4(5), 371-375.

[96] Thursky, K. A., \& Mahemoff, M. (2007). User-centered design techniques for a computerised antibiotic decision support system in an intensive care unit. Int J Med Inform, 76(10), 760-768. 
[97] Thursky, K. A., et al. (2006). Reduction of broad-spectrum antibiotic use with computerized decision support in an intensive care unit. Int J Qual Health Care, 18(3), 224-231.

[98] Mc Kinley, B. A., et al. (2011). Computer protocol facilitates evidence-based care of sepsis in the surgical intensive care unit. J Trauma, 70(5), 1153-1166, discussion 1166-7.

[99] Steurbaut, K., et al. (2012). COSARA: Integrated Service Platform for Infection Surveillance and Antibiotic Management in the ICU. J Med Syst. 
(C) 2012 The Author(s). Licensee IntechOpen. This is an open access article distributed under the terms of the Creative Commons Attribution 3.0 License, which permits unrestricted use, distribution, and reproduction in any medium, provided the original work is properly cited. 